\title{
Existence of flips and minimal models for 3 -folds in char $p$
}

\author{
Caucher Birkar
}

\begin{abstract}
We will prove the following results for 3 -fold pairs $(X, B)$ over an algebraically closed field $k$ of characteristic $p>5$ : $\log$ flips exist for $\mathbb{Q}$ factorial dlt pairs $(X, B)$; log minimal models exist for projective klt pairs $(X, B)$ with pseudo-effective $K_{X}+B$; the $\log$ canonical ring $R\left(K_{X}+B\right)$ is finitely generated for projective klt pairs $(X, B)$ when $K_{X}+B$ is a big $\mathbb{Q}$ divisor; semi-ampleness holds for a nef and big $\mathbb{Q}$-divisor $D$ if $D-\left(K_{X}+B\right)$ is nef and big and $(X, B)$ is projective klt; $\mathbb{Q}$-factorial dlt models exist for lc pairs $(X, B)$; terminal models exist for klt pairs $(X, B)$; ACC holds for lc thresholds; etc.
\end{abstract}

\section{Contents}

1. Introduction 2

2. Preliminaries

2.1. Contractions 7

2.2. Some notions related to divisors 7

2.3. The negativity lemma 7

2.4. Resolution of singularities 8

2.5. Pairs 8

2.6. Ample divisors on log smooth pairs 9

2.7. Models of pairs 9

2.8. Keel's results 9

3. Extremal rays and special kinds of LMMP 11

3.1. Extremal curve of a ray 11

3.2. Negative extremal rays 11

3.3. More on negative extremal rays 12

3.4. Extremal rays given by scaling. 13

$\begin{array}{ll}\text { 3.5. LMMP with scaling } & 13\end{array}$

3.6. Extremal rays given by a weak Zariski decomposition 14

3.7. LMMP using a weak Zariski decomposition 16

4. Adjunction 16

5. Special termination 19

5.1. Reduced components of boundaries of dlt pairs 20

5.3. Pl-extremal rays 20

5.4. Special termination 20

Date: July 13, 2018.

2010 MSC: 14E30. 
6. Existence of log flips 21

6.1. Divisorial and flipping extremal rays 22

6.2. Existence of generalized flips $\quad 22$

6.4. Proof of 1.1 in the projective case 24

7. Crepant models 24

7.1. Divisorial extremal rays 24

7.3. Projectivization and dlt models 25

7.6. Extraction of divisors and terminal models 26

8. Existence of log minimal models 27

8.1. Weak Zariski decompositions 27

8.2. From weak Zariski decompositions to minimal models 27

8.4. Proofs of 1.2 and $1.1 \quad 30$

9. The connectedness principle with applications to semi-ampleness 31

9.1. Connectedness 31

9.4. Semi-ampleness $\quad 35$

10. Finite generation and base point freeness 36

10.1. Finite generation 36

10.4. Base point freeness 40

10.5. Contractions 41

11. ACC for lc thresholds 41

11.1. ACC for lc thresholds on surfaces 41

11.4. Global ACC for surfaces 42

11.8. 3-folds 45

12. Non-big log divisors: proof of $1.11 \quad 45$

$\begin{array}{ll}\text { References } & 47\end{array}$

\section{INTRODUCTION}

We work over an algebraically closed field $k$ of characteristic (char) $p>0$. The pairs $(X, B)$ we consider in this paper always have $\mathbb{R}$-boundaries $B$ unless otherwise stated.

Higher dimensional birational geometry in char $p$ is still largely conjectural. Even the most basic problems such as base point freeness are not solved in general. Ironically though Mori's work on existence of rational curves which plays an important role in characteristic 0 uses reduction $\bmod p$ techniques. There are two reasons among others which have held back progress in char $p$ : resolution of singularities is not known and Kawamata-Viehweg vanishing fails. However, it was expected that one can work out most components of the minimal model program in dimension 3. This is because resolution of singularities is known in dimension 3 and many problems can be reduced to dimension 2 hence one can use special features of surface geometry.

On the positive side there has been some good progress toward understanding birational geometry in char $p$. People have tried to replace the characteristic 0 


\section{Existence of flips and minimal models for 3-folds in char $p$}

tools that fail in char $p$. For example, Keel [16] developed techniques for dealing with the base point free problem and semi-ampleness questions in general without relying on Kawamata-Viehweg vanishing type theorems. On the other hand, motivated by questions in commutative algebra, people have introduced Frobenius-singularities whose definition do not require resolution of singularities and they are very similar to singularities in characteristic 0 (cf. [24]).

More recently Hacon-Xu [13] proved the existence of flips in dimension 3 for pairs $(X, B)$ with $B$ having standard coefficients, that is, coefficients in $\mathfrak{S}=\left\{1-\frac{1}{n} \mid n \in \mathbb{N} \cup\{\infty\}\right\}$, and char $p>5$. From this they could derive existence of minimal models for 3 -folds with canonical singularities. In this paper, we rely on their results and ideas. The requirement $p>5$ has to do with the behavior of singularities on surfaces, eg a klt surface singularity over $k$ of char $p>5$ is strongly $F$-regular.

Log flips. Our first result is on the existence of flips.

Theorem 1.1. Let $(X, B)$ be a $\mathbb{Q}$-factorial dlt pair of dimension 3 over $k$ of char $p>5$. Let $X \rightarrow Z$ be a $K_{X}+B$-negative extremal flipping projective contraction. Then its flip exists.

The conclusion also holds if $(X, B)$ is klt but not necessarily $\mathbb{Q}$-factorial. This follows from the finite generation below (1.3). The theorem is proved in Section 6 when $X$ is projective. The quasi-projective case is proved in Section 8. We reduce the theorem to the case when $X$ is projective, $B$ has standard coefficients, and some component of $\lfloor B\rfloor$ is negative on the extremal ray: this case is $[13$, Theorem 4.12] which is one of the main results of that paper. A different approach is taken in [7] to prove 1.1 when $B$ has hyperstandard coefficients and $p \gg 0$ (these coefficients are of the form $\frac{n-1}{n}+\sum \frac{l_{i} b_{i}}{n}$ where $n \in \mathbb{N} \cup\{\infty\}$, $l_{i} \in \mathbb{Z}^{\geq 0}$ and $b_{i}$ are in some fixed DCC set).

To prove Theorem 1.1 we actually first prove the existence of generalized flips [13, after Theorem 5.6]. See Section 6 for more details.

Log minimal models. In [13, after Theorem 5.6], using generalized flips, a generalized $L M M P$ is defined which is used to show the existence of minimal models for varieties with canonical singularities (or for pairs with canonical singularities and "good" boundaries). Using weak Zariski decompositions as in [2], we construct log minimal models for klt pairs in general.

Theorem 1.2. Let $(X, B)$ be a klt pair of dimension 3 over $k$ of char $p>5$ and let $X \rightarrow Z$ be a projective contraction. If $K_{X}+B$ is pseudo-effective/ $Z$, then $(X, B)$ has a log minimal model over $Z$.

The theorem is proved in Section 8. Alternatively, one can apply the methods of [3] to construct log minimal models for lc pairs $(X, B)$ such that $K_{X}+B \equiv$ $M / Z$ for some $M \geq 0$. Note that when $X \rightarrow Z$ is a semi-stable fibration over a curve and $B=0$, the theorem was proved much earlier by Kawamata [14]. 
Remark on Mori fibre spaces. Let $(X, B)$ be a projective klt pair of dimension 3 over $k$ of char $p>5$ such that $K_{X}+B$ is not pseudo-effective. An important question is whether $(X, B)$ has a Mori fibre space. There is an ample $\mathbb{R}$-divisor $A \geq 0$ such that $K_{X}+B+A$ is pseudo-effective but $K_{X}+B+(1-\epsilon) A$ is not pseudo-effective for any $\epsilon>0$. Moreover, we may assume that $(X, B+A)$ is klt as well (9.2). By Theorem 1.2, $(X, B+A)$ has a log minimal model $\left(Y, B_{Y}+A_{Y}\right)$. Since $K_{Y}+B_{Y}+A_{Y}$ is not big, $K_{Y}+B_{Y}+A_{Y}$ is numerically trivial on some covering family of curves by [9](see also 1.11 below). Again by [9], there is a nef reduction map $Y \rightarrow T$ for $K_{Y}+B_{Y}+A_{Y}$ which is projective over the generic point of $T$. Although $Y \rightarrow T$ is not necessarily a Mori fibre space but in some sense it is similar.

Finite generation, base point freeness, and contractions. We will prove finite generation in the big case from which we can derive base point freeness and contractions of extremal rays in many cases. These are proved in Section 10 .

Theorem 1.3. Let $(X, B)$ be a klt pair of dimension 3 over $k$ of char $p>5$ and $X \rightarrow Z$ a projective contraction. Assume that $K_{X}+B$ is a $\mathbb{Q}$-divisor which is big/Z. Then the relative log canonical algebra $\mathcal{R}\left(K_{X}+B / Z\right)$ is finitely generated over $\mathcal{O}_{Z}$.

Assume that $Z$ is a point. If $K_{X}+B$ is not big, then $R\left(K_{X}+B / Z\right)$ is still finitely generated if $\kappa\left(K_{X}+B\right) \leq 1$. It remains to show the finite generation when $\kappa\left(K_{X}+B\right)=2$ : this can probably be reduced to dimension 2 using an appropriate canonical bundle formula, for example as in [9].

A more or less immediate consequence of the above finite generation is the following base point freeness.

Theorem 1.4. Let $(X, B)$ be a projective klt pair of dimension 3 over $k$ of char $p>5$ and $X \rightarrow Z$ a projective contraction where $B$ is a $\mathbb{Q}$-divisor. Assume that $D$ is a $\mathbb{Q}$-divisor such that $D$ and $D-\left(K_{X}+B\right)$ are both nef and big/Z. Then $D$ is semi-ample/Z.

Assume that $Z$ is a point. When $D-\left(K_{X}+B\right)$ is nef and big but $D$ is nef with numerical dimension $\nu(D)$ one or two, semi-ampleness of $D$ is proved in [9] under some restrictions on the coefficients.

Theorem 1.5. Let $(X, B)$ be a projective $\mathbb{Q}$-factorial dlt pair of dimension 3 over $k$ of char $p>5$, and $X \rightarrow Z$ a projective contraction. Let $R$ be a $K_{X}+B$ negative extremal ray $/ Z$. Assume that there is a nef and big/ $Z \mathbb{Q}$-divisor $N$ such that $N \cdot R=0$. Then $R$ can be contracted by a projective morphism.

Note that if $K_{X}+B$ is pseudo-effective/ $Z$, then for every $K_{X}+B$-negative extremal ray $R / Z$ there exists $N$ as in the theorem (see 3.3). Therefore such extremal rays can be contracted by projective morphisms.

Theorems 1.4 and 1.5 have been proved by $\mathrm{Xu}$ [30] independently and more or less at the same time but using a different approach. His proof also relies on 
our results on flips and minimal models.

Dlt and terminal models. The next two results are standard consequences of the LMMP (more precisely, of special termination). They are proved in Section 7.

Theorem 1.6. Let $(X, B)$ be an lc pair of dimension 3 over $k$ of char $p>5$. Then $(X, B)$ has a (crepant) $\mathbb{Q}$-factorial dlt model. In particular, if $(X, B)$ is klt, then $X$ has a $\mathbb{Q}$-factorialization by a small morphism.

The theorem was proved in [13, Theorem 6.1] for pairs with standard coefficients.

Theorem 1.7. Let $(X, B)$ be a klt pair of dimension 3 over $k$ of char $p>5$. Then $(X, B)$ has a (crepant) $\mathbb{Q}$-factorial terminal model.

The theorem was proved in [13, Theorem 6.1] for pairs with standard coefficients and canonical singularities.

The connectedness principle with applications to semi-ampleness. The next result concerns the Kollár-Shokurov connectedness principle. In characteristic 0 , the surface case was proved by Shokurov by taking a resolution and then calculating intersection numbers [26, Lemma 5.7] but the higher dimensional case was proved by Kollár by deriving it from the Kawamata-Viehweg vanishing theorem [20, Theorem 17.4].

Theorem 1.8. Let $(X, B)$ be a projective $\mathbb{Q}$-factorial pair of dimension 3 over $k$ of char $p>5$. Let $f: X \rightarrow Z$ be a birational contraction such that $-\left(K_{X}+B\right)$ is ample/Z. Then for any closed point $z \in Z$, the non-klt locus of $(X, B)$ is connected in any neighborhood of the fibre $X_{z}$.

The theorem is proved in Section 9. To prove it we use the LMMP rather than vanishing theorems. When $\operatorname{dim} X=2$, the theorem holds in a stronger form (see 9.3).

We will use the connectedness principle on surfaces to prove some semiampleness results on surfaces and 3-folds. Here is one of them:

Theorem 1.9. Let $(X, B+A)$ be a projective $\mathbb{Q}$-factorial dlt pair of dimension 3 over $k$ of char $p>5$. Assume that $A, B \geq 0$ are $\mathbb{Q}$-divisors such that $A$ is ample and $\left.\left(K_{X}+B+A\right)\right|_{\lfloor B\rfloor}$ is nef. Then $\left.\left(\bar{K}_{X}+B+A\right)\right|_{\lfloor B\rfloor}$ is semi-ample.

Note that if one could show that $\lfloor B\rfloor$ is semi-lc, then the result would follow from Tanaka [29]. In order to show that $\lfloor B\rfloor$ is semi-lc one needs to check that it satisfies the Serre condition $S_{2}$. In characteristic 0 this is a consequence of Kawamata-Viehweg vanishing (see Kollár [20, Corollary 17.5]). The $S_{2}$ condition can be used to glue sections on the various irreducible components of $\lfloor B\rfloor$. To prove the above semi-ampleness we instead use a result of Keel $[16$, Corollary 2.9] to glue sections. 
Log canonical thresholds. As in characteristic 0, we will derive the following result from existence of $\mathbb{Q}$-factorial dlt models and boundedness results on Fano surfaces.

Theorem 1.10. Suppose that $\Lambda \subseteq[0,1]$ and $\Gamma \subseteq \mathbb{R}$ are $D C C$ sets. Then the set

$$
\{\operatorname{lct}(M, X, B) \mid(X, B) \text { is lc of dimension } \leq 3\}
$$

satisfies the $A C C$ where $X$ is over $k$ with char $p>5$, the coefficients of $B$ belong to $\Lambda, M \geq 0$ is an $\mathbb{R}$-Cartier divisor with coefficients in $\Gamma$, and $\operatorname{lct}(M, X, B)$ is the lc threshold of $M$ with respect to $(X, B)$.

With some work it seems that using the above ACC one can actually prove termination for those lc pairs $(X, B)$ of dimension 3 such that $K_{X}+B \equiv M$ for some $M \geq 0$ following the ideas in [4]. But we will not pursue this here.

Numerically trivial family of curves in the non-big case. We will also give a somewhat different proof of the following result which was proved by CasciniTanaka-Xu [9] in char $p$. This was also proved independently by MKernan much earlier but unpublished. He informed us that his proof was inspired by $[17]$.

Theorem 1.11. Assume that $X$ is a normal projective variety of dimensiond over an algebraically closed field (of any characteristic), and that $B, A \geq 0$ are $\mathbb{R}$-divisors. Moreover, suppose $A$ is nef and big and $D=K_{X}+B+A$ is nef. If $D^{d}=0$, then for each general closed point $x \in X$ there is a rational curve $L_{x}$ passing through $x$ with $D \cdot L_{x}=0$.

The theorem is independent of the rest of this paper. Its proof is an application of the bend and break theorem.

Some remarks about this paper. In writing this paper we have tried to give as much details as possible even if the arguments are very similar to the characteristic 0 case. This is for convenience, future reference, and to avoid any unpleasant surprise having to do with positive characteristic. The main results are proved in the following order: 1.1 in the projective case, 1.6, 1.7, 1.2, 1.1 in general, $1.8,1.9,1.3,1.4,1.5,1.10$, and 1.11 .

Acknowledgements. Part of this work was done when I visited National Taiwan University in September 2013 with the support of the Mathematics Division (Taipei Office) of the National Center for Theoretical Sciences. The visit was arranged by Jungkai A. Chen. I would like to thank them for their hospitality. This work was partially supported by a Leverhulme grant. I would also like to thank Paolo Cascini, Christopher Hacon, Janos Kollár, James McKernan, Burt Totaro and Chenyang $\mathrm{Xu}$ for their comments and suggestions. Finally I would like to thank the referee for valuable corrections and suggestions. 


\section{Existence of flips and minimal models for 3-folds in char $p$}

\section{Preliminaries}

We work over an algebraically closed field $k$ of characteristic $p>0$ fixed throughout the paper unless stated otherwise.

2.1. Contractions. A contraction $f: X \rightarrow Z$ of algebraic spaces over $k$ is a proper morphism such that $f_{*} \mathcal{O}_{X}=\mathcal{O}_{Z}$. When $X, Z$ are quasi-projective varieties over $k$ and $f$ is projective, we refer to $f$ as a projective contraction to avoid confusion.

Let $f: X \rightarrow Z$ be a projective contraction of normal varieties. We say $f$ is extremal if the relative Kleiman-Mori cone of curves $\overline{N E}(X / Z)$ is onedimensional. Such a contraction is a divisorial contraction if it is birational and it contracts some divisor. It is called a small contraction if it is birational and it contracts some subvariety of codimension $\geq 2$ but no divisors.

Let $f: X \rightarrow Z$ be a small contraction and $D$ an $\mathbb{R}$-Cartier divisor such that $-D$ is ample $/ Z$. We refer to $f$ as a $D$-flipping contraction or just a flipping contraction for short. We say the $D$-flip of $f$ exists if there is a small contraction $X^{+} \rightarrow Z$ such that the birational transform $D^{+}$is ample/Z.

2.2. Some notions related to divisors. Let $X$ be a normal projective variety over $k$ and $L$ a nef $\mathbb{R}$-Cartier divisor. We define $L^{\perp}:=\{\alpha \in \overline{N E}(X) \mid L \cdot \alpha=0\}$. This is an extremal face of $\overline{N E}(X)$ cut out by $L$.

Let $f: X \rightarrow Z$ be a projective morphism of normal varieties over $k$, and let $D$ be an $\mathbb{R}$-divisor on $X$. We define the algebra of $D$ over $Z$ as $\mathcal{R}(D / Z)=$ $\bigoplus_{m \in \mathbb{Z} \geq 0} f_{*} \mathcal{O}_{X}(\lfloor m D\rfloor)$. When $Z$ is a point we denote the algebra by $R(D)$. When $D=K_{X}+B$ for a pair $(X, B)$ we call the algebra the log canonical algebra of $(X, B)$ over $Z$.

Now let $\phi: X \rightarrow Y$ be a birational map of normal projective varieties over $k$ whose inverse does not contract divisors. Let $D$ be an $\mathbb{R}$-Cartier divisor on $X$ such that $D_{Y}:=\phi_{*} D$ is $\mathbb{R}$-Cartier too. We say that $\phi$ is $D$-negative if there is a common resolution $f: W \rightarrow X$ and $g: W \rightarrow Y$ such that $f^{*} D-g^{*} D_{Y}$ is effective and exceptional $/ Y$, and its support contains the birational transform of all the prime divisors on $X$ which are contracted/ $Y$.

2.3. The negativity lemma. The negativity lemma states that if $f: Y \rightarrow X$ is a projective birational contraction of normal quasi-projective varieties over $k$ and $D$ is an $\mathbb{R}$-Cartier divisor on $Y$ such that $-D$ is nef $/ X$ and $f_{*} D \geq 0$, then $D \geq 0$ (since this is a local statement over $X$, it also holds if we assume $X$ is an algebraic space and $f$ is proper). See [26, Lemma 1.1] for the characteristic 0 case. The proof there also works in char $p>0$ and we reproduce it for convenience. Assume that the lemma does not hold. We reduce the problem to the surface case. Let $P$ be the image of the negative components of $D$. If $\operatorname{dim} P>0$, we take a general hypersurface section $H$ on $X$, let $G$ be the normalization of the birational transform of $H$ on $Y$ and reduce the problem to the contraction $G \rightarrow H$ and the divisor $\left.D\right|_{G}$. But if $\operatorname{dim} X>2$ and $\operatorname{dim} P=0$, we take a general hypersurface section $G$ on $Y$, let $H$ be the normalization of $f(G)$, and reduce the problem to the induced contraction $G \rightarrow H$ and divisor 
$\left.D\right|_{G}$. So we can reduce the problem to the case when $X, Y$ are surfaces, $P$ is just one point, and $f$ is an isomorphism over $X \backslash\{P\}$. Taking a resolution enables us to assume $Y$ is smooth. Now let $E \geq 0$ be a divisor whose support is equal to the exceptional locus of $f$ and such that $-E$ is nef $/ X$ : pick a Cartier divisor $L \geq 0$ passing through $P$ and write $f^{*} L=L^{\sim}+E$ where $L^{\sim}$ is the birational transform of $L$; then $E$ satisfies the requirements. Let $e$ be the smallest number such that $D+e E \geq 0$. Now there is a component $C$ of $E$ whose coefficient in $D+e E$ is zero and that $C$ intersects $\operatorname{Supp}(D+e E)$. But then $(D+e E) \cdot C>0$, a contradiction.

2.4. Resolution of singularities. Let $X$ be a quasi-projective variety of dimension $\leq 3$ over $k$ and $P \subset X$ a closed subset. Assume that there is an open set $U \subset X$ such that $P \cap U$ is a divisor with simple normal crossing (snc) singularities. Then there is a log resolution of $X, P$ which is an isomorphism over $U$, that is, there is a projective birational morphism $f: Y \rightarrow X$ such that the union of the exceptional locus of $f$ and the birational transform of $P$ is an snc divisor, and $f$ is an isomorphism over $U$. This follows from Cutkosky [12, Theorems 1.1, 1.2, 1.3] when $k$ has char $p>5$, and from Cossart-Piltant [11, Theorems 4.1, 4.2][10, Theorem] in general (see also [13, Theorem 2.1]).

2.5. Pairs. A pair $(X, B)$ consists of a normal quasi-projective variety $X$ over $k$ and an $\mathbb{R}$-boundary $B$, that is an $\mathbb{R}$-divisor $B$ on $X$ with coefficients in $[0,1]$, such that $K_{X}+B$ is $\mathbb{R}$-Cartier. When $B$ has rational coefficients we say $B$ is a $\mathbb{Q}$-boundary or say $B$ is rational. We say that $(X, B)$ is $\log$ smooth if $X$ is smooth and Supp $B$ has simple normal crossing singularities.

Let $(X, B)$ be a pair. For a prime divisor $D$ on some birational model of $X$ with a nonempty centre on $X, a(D, X, B)$ denotes the log discrepancy which is defined by taking a projective birational morphism $f: Y \rightarrow X$ from a normal variety containing $D$ as a prime divisor and putting $a(D, X, B)=1-b$ where $b$ is the coefficient of $D$ in $B_{Y}$ and $K_{Y}+B_{Y}=f^{*}\left(K_{X}+B\right)$.

As in characteristic 0 , we can define various types of singularities using log discrepancies. Let $(X, B)$ be a pair. We say that the pair is log canonical or lc for short (resp. Kawamata log terminal or klt for short) if $a(D, X, B) \geq 0$ (resp. $a(D, X, B)>0$ ) for any prime divisor $D$ on birational models of $X$. An $l c$ centre of $(X, B)$ is the image in $X$ of a $D$ with $a(D, X, B)=0$. The pair $(X, B)$ is terminal if $a(D, X, B)>1$ for any prime divisor $D$ on birational models of $X$ which is exceptional/ $X$ (such pairs are sometime called terminal in codimension $\geq 2)$. On the other hand, we say that $(X, B)$ is $d l t$ if there is a closed subset $P \subset X$ such that $(X, B)$ is $\log$ smooth outside $P$ and no lc centre of $(X, B)$ is inside $P$. In particular, the lc centres of $(X, B)$ are exactly the components of $S_{1} \cap \cdots \cap S_{r}$ where $S_{i}$ are among the components of $\lfloor B\rfloor$. Moreover, there is a $\log$ resolution $f: Y \rightarrow X$ of $(X, B)$ such that $a(D, X, B)>0$ for any prime divisor $D$ on $Y$ which is exceptional/ $X$, eg take a $\log$ resolution $f$ which is an isomorphism over $X \backslash P$. Finally, we say that $(X, B)$ is $p l t$ if it is dlt and each connected component of $\lfloor B\rfloor$ is irreducible. In particular, the only lc centres of $(X, B)$ are the components of $\lfloor B\rfloor$. 
2.6. Ample divisors on $\log$ smooth pairs. Let $(X, B)$ be a projective log smooth pair over $k$ and let $A$ be an ample $\mathbb{Q}$-divisor. We will argue that there is $A^{\prime} \sim_{\mathbb{Q}} A$ such that $A^{\prime} \geq 0$ and that $\left(X, B+A^{\prime}\right)$ is log smooth. The argument was suggested to us by several people independently. We may assume that $B$ is reduced. Let $S_{1}, \ldots, S_{r}$ be the components of $B$ and let $\mathcal{S}$ be the set of the components of $S_{i_{1}} \cap \cdots \cap S_{i_{n}}$ for all the choices $\left\{i_{1}, \ldots, i_{n}\right\} \subseteq\{1, \cdots, r\}$. By Bertini's theorem, there is a sufficiently divisible integer $l>0$ such that for any $T \in \mathcal{S}$, a general element of $|l A|_{T} \mid$ is smooth. Since $l A$ is sufficiently ample, such general elements are restrictions of general elements of $|l A|$. Therefore, we can choose a general $G \sim l A$ such that $G$ is smooth and $\left.G\right|_{T}$ is smooth for any $T \in \mathcal{S}$. This means that $(X, B+G)$ is $\log$ smooth. Now let $A^{\prime}=\frac{1}{l} G$.

2.7. Models of pairs. Let $(X, B)$ be a pair and $X \rightarrow Z$ a projective contraction over $k$. A pair $\left(Y, B_{Y}\right)$ with a projective contraction $Y \rightarrow Z$ and a birational map $\phi: X \rightarrow Y / Z$ is a $\log$ birational model of $(X, B)$ if $B_{Y}$ is the sum of the birational transform of $B$ and the reduced exceptional divisor of $\phi^{-1}$. We say that $\left(Y, B_{Y}\right)$ is a weak lc model of $(X, B)$ over $Z$ if in addition

(1) $K_{Y}+B_{Y}$ is nef $/ Z$.

(2) for any prime divisor $D$ on $X$ which is exceptional $Y$, we have

$$
a(D, X, B) \leq a\left(D, Y, B_{Y}\right)
$$

And we call $\left(Y, B_{Y}\right)$ a log minimal model of $(X, B)$ over $Z$ if in addition

(3) $\left(Y, B_{Y}\right)$ is $\mathbb{Q}$-factorial dlt,

(4) the inequality in (2) is strict.

When $K_{X}+B$ is big/ $Z$, the lc model of $(X, B)$ over $Z$ is a weak lc model $\left(Y, B_{Y}\right)$ over $Z$ with $K_{Y}+B_{Y}$ ample $/ Z$.

On the other hand, a $\log$ birational model $\left(Y, B_{Y}\right)$ of $(X, B)$ is called a Mori fibre space of $(X, B)$ over $Z$ if there is a $K_{Y}+B_{Y}$-negative extremal projective contraction $Y \rightarrow T / Z$, and if for any prime divisor $D$ on birational models of $X$ we have

$$
a(D, X, B) \leq a\left(D, Y, B_{Y}\right)
$$

with strict inequality if $D \subset X$ and if it is exceptional $/ Y$,

Note that the above definitions are slightly different from the traditional definitions. However, if $(X, B)$ is plt (hence also klt) the definitions coincide.

Let $(X, B)$ be an lc pair over $k$. A $\mathbb{Q}$-factorial dlt pair $\left(Y, B_{Y}\right)$ is a $\mathbb{Q}$-factorial dlt model of $(X, B)$ if there is a projective birational morphism $f: Y \rightarrow X$ such that $K_{Y}+B_{Y}=f^{*}\left(K_{X}+B\right)$ and such that every exceptional prime divisor of $f$ has coefficient 1 in $B_{Y}$. On the other hand, when $(X, B)$ is klt, a pair $\left(Y, B_{Y}\right)$ with terminal singularities is a terminal model of $(X, B)$ if there is a projective birational morphism $f: Y \rightarrow X$ such that $K_{Y}+B_{Y}=f^{*}\left(K_{X}+B\right)$.

2.8. Keel's results. We recall some of the results of Keel which will be used in this paper. For a nef $\mathbb{Q}$-Cartier divisor $L$ on a projective scheme $X$ over $k$, 
the exceptional locus $\mathbb{E}(L)$ is the union of those positive-dimensional integral subschemes $Y \subseteq X$ such that $\left.L\right|_{Y}$ is not big, i.e. $\left(\left.L\right|_{Y}\right)^{\operatorname{dim} Y}=0$. By [8], $\mathbb{E}(L)$ coincides with the augmented base locus $\mathbf{B}_{+}(L)$. We say $L$ is endowed with a map $f: X \rightarrow V$, where $V$ is an algebraic space over $k$, if: an integral subscheme $Y$ is contracted by $f$ (i.e. $\operatorname{dim} Y>\operatorname{dim} f(Y)$ ) if and only if $\left.L\right|_{Y}$ is not big.

Theorem 2.9 ([16, 1.9]). Let $X$ be a projective scheme over $k$ and $L$ a nef $\mathbb{Q}$-Cartier divisor on $X$. Then

- $L$ is semi-ample if and only if $\left.L\right|_{\mathbb{E}(L)}$ is semi-ample;

- $L$ is endowed with a map if and only if $\left.L\right|_{\mathbb{E}(L)}$ is endowed with a map.

The theorem does not hold if $k$ is of characteristic 0 . When $\left.L\right|_{\mathbb{E}(L)} \equiv 0$, then $\left.L\right|_{\mathbb{E}(L)}$ is automatically endowed with the constant map $\mathbb{E}(L) \rightarrow$ pt hence $L$ is endowed with a map. This is particularly useful for studying 3-folds because it is often not difficult to show that $\left.L\right|_{\mathbb{E}(L)}$ is endowed with a map, eg when $\operatorname{dim} \mathbb{E}(L)=1$.

Theorem 2.10 ([16, 0.5]). Let $(X, B)$ be a projective $\mathbb{Q}$-factorial pair of dimension 3 over $k$ with $B$ a $\mathbb{Q}$-divisor. Assume that $A$ is an ample $\mathbb{Q}$-divisor such that $L=K_{X}+B+A$ is nef and big. Then $L$ is endowed with a map.

In particular, when $L^{\perp}$ is an extremal ray, then we can contract $R$ to an algebraic space by the map associated to $L$. Thus such an extremal ray is generated by the class of some curve.

We also recall the following cone theorems which we will use repeatedly in Section 3. Note that these theorems (as well as 2.10) do not assume singularities to be lc.

Theorem 2.11 ([16, 0.6]). Let $(X, B)$ be a projective $\mathbb{Q}$-factorial pair of dimension 3 over $k$ with $B$ a $\mathbb{Q}$-divisor. Assume that $K_{X}+B \sim_{\mathbb{Q}} M$ for some $M \geq 0$. Then there is a countable number of curves $\Gamma_{i}$ such that

- $\overline{N E}(X)=\overline{N E}(X)_{K_{X}+B \geq 0}+\sum_{i} \mathbb{R}\left[\Gamma_{i}\right]$,

- all but finitely many of the $\Gamma_{i}$ are rational curves satisfying $-3 \leq\left(K_{X}+\right.$ $B) \cdot \Gamma_{i}<0$, and

- the rays $\mathbb{R}\left[\Gamma_{i}\right]$ do not accumulate inside $\overline{N E}(X)_{K_{X}+B<0}$.

Theorem 2.12 ([16, 5.5.2]). Let $(X, B)$ be a projective $\mathbb{Q}$-factorial pair of dimension 3 over $k$. Assume that

$$
L=K_{X}+B+H \sim_{\mathbb{R}} A+M
$$

is nef where $H, A$ are ample $\mathbb{R}$-divisors, and $M \geq 0$. Then any extremal ray of $L^{\perp}$ is generated by some curve $\Gamma$ such that either

- $\Gamma$ is a component of the singular locus of $B+M$ union with the singular locus of $X$, or

- $\Gamma$ is a rational curve satisfying $-3 \leq\left(K_{X}+B\right) \cdot \Gamma<0$.

Remark 2.13 Let $(X, B)$ a projective lc pair of dimension 3 over $k$ with $B$ a $\mathbb{Q}$-boundary, and $H$ an ample $\mathbb{Q}$-divisor. Assume that $L=K_{X}+B+H$ is nef 


\section{Existence of flips and minimal models for 3-folds in char $p \quad 11$}

and big. Moreover, suppose that each connected component of $\mathbb{E}(L)$ is inside some normal irreducible component $S$ of $\lfloor B\rfloor$. Then $\left.L\right|_{S}$ is semi-ample for such components (cf. [28]) hence $\left.L\right|_{\mathbb{E}(L)}$ is semi-ample and this in turn implies that $L$ is semi-ample by Theorem 2.9 .

\section{Extremal RAYS AND SPECIAL Kinds OF LMMP}

As usual the varieties and algebraic spaces in this section are defined over $k$ of $\operatorname{char} p>0$.

3.1. Extremal curve of a ray. Let $X$ be a projective variety and $H$ a fixed ample Cartier divisor. Let $R$ be a ray of $\overline{N E}(X)$ which is generated by some curve $\Gamma$. Assume that

$$
H \cdot \Gamma=\min \{H \cdot C \mid C \text { generates } R\}
$$

In this case, we say $\Gamma$ is an extremal curve of $R$ (in practice we do not mention $H$ and assume that it is already fixed). Let $C$ be any other curve generating $R$. Assume that $D \cdot R<0$ for some $\mathbb{R}$-Cartier divisor $D$. Since $\Gamma$ and $C$ both generate $R$,

hence

$$
\frac{D \cdot C}{H \cdot C}=\frac{D \cdot \Gamma}{H \cdot \Gamma}
$$

which implies that

$$
D \cdot \Gamma=D \cdot C\left(\frac{H \cdot \Gamma}{H \cdot C}\right) \geq D \cdot C
$$

$$
D \cdot \Gamma=\max \{D \cdot C \mid C \text { generates } R\}
$$

3.2. Negative extremal rays. Let $(X, B)$ be a projective $\mathbb{Q}$-factorial pair of dimension 3. Let $R$ be a $K_{X}+B$-negative extremal ray. Assume that there is a boundary $\Delta$ such that $K_{X}+\Delta$ is pseudo-effective and $\left(K_{X}+\Delta\right) \cdot R<0$. By adding a small ample divisor and perturbing the coefficients we can assume that $\Delta$ is rational and that $K_{X}+\Delta$ is big. Then by Theorem $2.11, R$ is generated by some extremal curve and $R$ is an isolated extremal ray of $\overline{N E}(X)$.

Now assume that $K_{X}+B$ is pseudo-effective and let $A$ be an ample $\mathbb{R}$-divisor. Then for any $\epsilon>0$, there are only finitely many $K_{X}+B+\epsilon A$-negative extremal rays: assume that this is not the case; then we can find a $\mathbb{Q}$-boundary $\Delta$ such that $K_{X}+\Delta$ is big and

$$
K_{X}+B+\epsilon A \sim_{\mathbb{R}} K_{X}+\Delta+G
$$

where $G$ is ample; so there are also infinitely many $K_{X}+\Delta$-negative extremal rays; but $K_{X}+\Delta$ is big hence by Theorem 2.11 all but finitely many of the $K_{X}+\Delta$-negative extremal rays are generated by extremal curves $\Gamma$ with $-3 \leq$ $\left(K_{X}+\Delta\right) \cdot \Gamma<0$; if $\left(K_{X}+B+\epsilon A\right) \cdot \Gamma<0$, then $G \cdot \Gamma \leq 3$; since $G$ is ample, there can be only finitely many such $\Gamma$ up to numerical equivalence.

Let $R$ be a $K_{X}+B$-negative extremal ray where $K_{X}+B$ is not necessarily pseudo-effective. But assume that there is a pseudo-effective $K_{X}+\Delta$ with $\left(K_{X}+\Delta\right) \cdot R<0$. By the remarks above we may assume $\Delta$ is rational, $K_{X}+\Delta$ big, and that there are only finitely many $K_{X}+\Delta$-negative extremal rays. 
Therefore, we can find an ample $\mathbb{Q}$-divisor $H$ such that $L=K_{X}+\Delta+H$ is nef and big and $L^{\perp}=R$. That is, $L$ is a supporting divisor of $R$. Moreover, $R$ can be contracted to an algebraic space, by Theorem 2.10. More precisely, there is a contraction $X \rightarrow V$ to an algebraic space such that it contracts a curve $C$ if and only if $L \cdot C=0$ if and only if the class $[C] \in R$.

3.3. More on negative extremal rays. Let $(X, B)$ be a projective $\mathbb{Q}$-factorial pair of dimension 3. Let $\mathcal{C} \subset \overline{N E}(X)$ be one of the following:

(1) $\mathcal{C}=\overline{N E}(X / Z)$ for a given projective contraction $X \rightarrow Z$ such that $K_{X}+B \equiv P+M / Z$ where $P$ is nef $/ Z$ and $M \geq 0$ (this is a weak Zariski decomposition; see 8.1); or

(2) $\mathcal{C}=N^{\perp}$ for some nef and big $\mathbb{Q}$-divisor $N$;

We will show that in both cases, each $K_{X}+B$-negative extremal ray $R$ of $\mathcal{C}$ is generated by an extremal curve $\Gamma$, and for all but finitely many of those rays we have $-3 \leq\left(K_{X}+B\right) \cdot \Gamma<0$.

We first deal with case (1). Fix a $K_{X}+B$-negative extremal ray $R$ of $\mathcal{C}$. By replacing $P$ we can assume that $K_{X}+B=P+M$. Let $A$ be an ample $\mathbb{R}$-divisor and $T$ be the pullback of a sufficiently ample divisor on $Z$ so that $K_{X}+B+A+T$ is big and $\left(K_{X}+B+A+T\right) \cdot R<0$. By 3.2, there is a nef and big $\mathbb{Q}$-divisor $L$ with $L^{\perp}=R$. Moreover, we may assume that if $l \gg 0$, then

$$
Q_{1}:=K_{X}+B+T+l L+A
$$

is nef and big and $Q_{1}^{\perp}=R$. By construction, $T+l L+A$ is ample, $P+T+l L+A$ is also ample, and

$$
K_{X}+B+T+l L+A=P+T+l L+A+M
$$

Therefore, by Theorem 2.12, $R$ is generated by some curve $\Gamma$ satisfying $-3 \leq$ $\left(K_{X}+B\right) \cdot \Gamma<0$ or $R$ is generated by some curve in the singular locus of $B+M$ or $X$. There are only finitely many possibilities in the latter case. The claim then follows.

Now we deal with case (2). Fix a $K_{X}+B$-negative extremal ray $R$ of $\mathcal{C}$. Since $N$ is nef and big, for some $n>0$,

$$
K_{X}+B+n N \sim_{\mathbb{R}} G+S
$$

where $G$ is ample and $S \geq 0$. By 3.2, there is a nef and big $\mathbb{Q}$-divisor $L$ with $L^{\perp}=R$. Moreover, for some $l \gg 0$ and some ample $\mathbb{R}$-divisor $A$,

$$
Q_{2}:=K_{X}+B+n N+l L+A
$$

is nef and big with $Q_{2}^{\perp}=R$. Now, $n N+l L+A$ is ample, $G+l L+A$ is ample, and

$$
K_{X}+B+n N+l L+A \sim_{\mathbb{R}} G+l L+A+S
$$

Therefore, by Theorem 2.12, $R$ is generated by some curve $\Gamma$ satisfying $-3 \leq$ $\left(K_{X}+B\right) \cdot \Gamma<0$ or $R$ is generated by some curve in the singular locus of $B+S$ or $X$. There are only finitely many possibilities in the latter case. The claim then follows. 


\section{Existence of flips and minimal models for 3-folds in char $p$}

13

Assume that $R$ is a $K_{X}+B$-negative extremal ray of $\mathcal{C}$, in either case. Then the above arguments show that there is a $\mathbb{Q}$-boundary $\Delta$ and an ample $\mathbb{Q}$ divisor $H$ such that $K_{X}+\Delta$ is big, $\left(K_{X}+\Delta\right) \cdot R<0$, and $L=K_{X}+\Delta+H$ is nef and big with $L^{\perp}=R$. Therefore, as in 3.2, $R$ can be contracted via a contraction $X \rightarrow V$ to an algebraic space. Moreover, if $B$ is rational, then we can find an ample $\mathbb{Q}$-divisor $H^{\prime}$ such that $L^{\prime}=K_{X}+B+H^{\prime}$ is nef and big and again $L^{\prime \perp}=R$.

3.4. Extremal rays given by scaling. Let $(X, B)$ be a projective $\mathbb{Q}$-factorial pair of dimension 3. Assume that either $\mathcal{C}=\overline{N E}(X / Z)$ for some projective contraction $X \rightarrow Z$ such that $K_{X}+B \equiv M / Z$ for some $M \geq 0$, or $\mathcal{C}=N^{\perp}$ for some nef and big $\mathbb{Q}$-divisor $N$. In addition assume that $(X, B+C)$ is a pair for some $C \geq 0$ and that $K_{X}+B+C$ is nef on $\mathcal{C}$, that is, $\left(K_{X}+B+C\right) \cdot R \geq 0$ for every extremal ray $R$ of $\mathcal{C}$. Let

$$
\lambda=\inf \left\{t \geq 0 \mid K_{X}+B+t C \text { is nef on } \mathcal{C}\right\}
$$

Then we will see that either $\lambda=0$ or there is an extremal ray $R$ of $\mathcal{C}$ such that $\left(K_{X}+B+\lambda C\right) \cdot R=0$ and $\left(K_{X}+B\right) \cdot R<0$. Assume $\lambda>0$. If the claim is not true, then there exist a sequence of numbers $t_{1}<t_{2}<\cdots$ approaching $\lambda$ and extremal rays $R_{i}$ of $\mathcal{C}$ such that $\left(K_{X}+B+t_{i} C\right) \cdot R_{i}=0$ and $\left(K_{X}+B\right) \cdot R_{i}<0$.

First assume that $\mathcal{C}=N^{\perp}$ for some nef and big $\mathbb{Q}$-divisor $N$. We can write a finite sum $K_{X}+B=\sum_{j} r_{j}\left(K_{X}+B_{j}\right)$ where $r_{j} \in(0,1], \sum r_{j}=1$, and $\left(X, B_{j}\right)$ are pairs with $B_{j}$ being rational. By 3.3, we may assume that each $R_{i}$ is generated by some extremal curve $\Gamma_{i}$ with $-3 \leq\left(K_{X}+B_{j}\right) \cdot \Gamma_{i}$ for each $j$. This implies that there are only finitely many possibilities for the numbers $\left(K_{X}+B\right) \cdot \Gamma_{i}$. A similar reasoning shows that there are only finitely many possibilities for the numbers $\left(K_{X}+B+\frac{\lambda}{2} C\right) \cdot \Gamma_{i}$ hence there are also only finitely many possibilities for the numbers $C \cdot \Gamma_{i}$. But then this implies that there are finitely many $t_{i}$, a contradiction.

Now assume that $\mathcal{C}=\overline{N E}(X / Z)$ for some projective contraction $X \rightarrow Z$ such that $K_{X}+B \equiv M / Z$ for some $M \geq 0$. Then we can write $K_{X}+B=$ $\sum_{j} r_{j}\left(K_{X}+B_{j}\right)$ and $M=\sum_{j} r_{j} M_{j}$ where $r_{j} \in(0,1], \sum r_{j}=1,\left(X, B_{j}\right)$ are pairs with $B_{j}$ being rational, $K_{X}+B_{j} \equiv M_{j} / Z$, and $M_{j} \geq 0$. To find such a decomposition we argue as in [6, pages 96-97]. Let $V$ and $W$ be the $\mathbb{R}$-vector spaces generated by the components of $B$ and $M$ respectively. For a vector $v \in V$ (resp. $w \in W$ ) we denote the corresponding $\mathbb{R}$-divisor by $B_{v}$ (resp. $M_{w}$ ). Let $F$ be the set of those $(v, w) \in V \times W$ such that $\left(X, B_{v}\right)$ is a pair, $M_{w} \geq 0$, and $K_{X}+B_{v} \equiv M_{w} / Z$. Then $F$ is defined by a finite number of linear equalities and inequalities with rational coefficients. If $B=B_{v_{0}}$ and $M=M_{w_{0}}$ are the given divisors, then $\left(v_{0}, w_{0}\right) \in F$ hence it belongs to some polytope in $F$ with rational vertices. The vertices of the polytope give the $B_{j}, M_{j}$. The rest of the proof is as in the last paragraph.

3.5. LMMP with scaling. Let $(X, B)$ be a projective $\mathbb{Q}$-factorial pair of dimension 3. Assume that either $\mathcal{C}=\overline{N E}(X / Z)$ for some projective contraction $X \rightarrow Z$ such that $K_{X}+B \equiv M / Z$ for some $M \geq 0$, or $\mathcal{C}=N^{\perp}$ for some nef 
and big $\mathbb{Q}$-divisor $N$. In addition assume that $(X, B+C)$ is a pair for some $C \geq 0$ and that $K_{X}+B+C$ is nef on $\mathcal{C}$.

If $K_{X}+B$ is not nef on $\mathcal{C}$, by 3.4 , there is an extremal ray $R$ of $\mathcal{C}$ such that $\left(K_{X}+B+\lambda C\right) \cdot R=0$ and $\left(K_{X}+B\right) \cdot R<0$ where $\lambda$ is the smallest number such that $K_{X}+B+\lambda C$ is nef on $\mathcal{C}$. Assume that $R$ can be contracted by a projective morphism. The contraction is birational because $L \cdot R=0$ for some nef and big $\mathbb{Q}$-Cartier divisor $L$ (see 3.3). Assume that $X \rightarrow X^{\prime}$ is the corresponding divisorial contraction or flip, and assume that $X^{\prime}$ is $\mathbb{Q}$-factorial. Let $\mathcal{C}^{\prime}$ be the cone given by $\mathcal{C}^{\prime}=\overline{N E}\left(X^{\prime} / Z\right)$ or $\mathcal{C}^{\prime}=\left(N^{\prime}\right)^{\perp}$ corresponding to the above cases. Let $\lambda^{\prime}$ be the smallest nonnegative number such that $K_{X^{\prime}}+B^{\prime}+\lambda^{\prime} C^{\prime}$ is nef on $\mathcal{C}^{\prime}$. If $\lambda^{\prime}>0$, then there is an extremal ray $R^{\prime}$ of $\mathcal{C}^{\prime}$ such that $\left(K_{X^{\prime}}+B^{\prime}+\lambda^{\prime} C^{\prime}\right) \cdot R^{\prime}=0$ and $\left(K_{X^{\prime}}+B^{\prime}\right) \cdot R^{\prime}<0$. Assume that $R^{\prime}$ can be contracted and so on. Assuming that all the necessary ingredients exist, the process gives a special kind of LMMP which we may refer to as $L M M P / \mathcal{C}$ on $K_{X}+B$ with scaling of $C$. Note that $\lambda \geq \lambda^{\prime} \geq \ldots$

If $\mathcal{C}=\overline{N E}(X / Z)$, we also refer to the above LMMP as the LMMP/Z on $K_{X}+B$ with scaling of $C$. If $\mathcal{C}=N^{\perp}$, and if $N$ is endowed with a map $X \rightarrow V$ to an algebraic space, we refer to the above LMMP as the LMMP/ $V$ on $K_{X}+B$ with scaling of $C$.

In practice, when we run an LMMP with scaling, $(X, B)$ is $\mathbb{Q}$-factorial dlt and each extremal ray in the process intersects some component of $\lfloor B\rfloor$ negatively. In particular, such rays can be contracted by projective morphisms and the $\mathbb{Q}$-factorial property is preserved by the LMMP (see 5.3). If the required flips exist then the LMMP terminates by special termination (see 5.4).

3.6. Extremal rays given by a weak Zariski decomposition. Let $(X, B)$ be a projective $\mathbb{Q}$-factorial pair of dimension 3 and $X \rightarrow Z$ a projective contraction such that

(1) $K_{X}+B \equiv P+M / Z, P$ is nef $/ Z, M \geq 0$, and

(2) Supp $M \subseteq\lfloor B\rfloor$.

Let

$$
\mu=\sup \{t \in[0,1] \mid P+t M \text { is nef } / Z\} .
$$

Assume that $\mu<1$. We will show that there is an extremal ray $R / Z$ such that $\left(K_{X}+B\right) \cdot R<0$ and $(P+\mu M) \cdot R=0$.

Replacing $P$ with $P+\mu M$ we may assume that $\mu=0$. Then by definition of $\mu, P+\epsilon^{\prime} M$ is not nef $/ Z$ for any $\epsilon^{\prime}>0$. In particular, for any $\epsilon^{\prime}>0$ there is a $K_{X}+B$-negative extremal ray $R / Z$ such that $\left(P+\epsilon^{\prime} M\right) \cdot R<0$ but $(P+\epsilon M) \cdot R=0$ for some $\epsilon \in\left[0, \epsilon^{\prime}\right)$. If there is no $K_{X}+B$-negative extremal ray $R / Z$ such that $P \cdot R=0$, then there is an infinite strictly decreasing sequence of sufficiently small positive real numbers $\epsilon_{i}$ and $K_{X}+B$-negative extremal rays $R_{i} / Z$ such that $\lim _{i \rightarrow \infty} \epsilon_{i}=0$ and $\left(P+\epsilon_{i} M\right) \cdot R_{i}=0$.

We may assume that for each $i$, there is an extremal curve $\Gamma_{i}$ generating $R_{i}$ such that $-3 \leq\left(K_{X}+B\right) \cdot \Gamma_{i}<0$ (see 3.3). Since Supp $M \subseteq\lfloor B\rfloor$, there is a small $\delta>0$ such that $\left(K_{X}+B-\delta M\right) \cdot \Gamma_{i}<0$ for each $i, B-\delta M \geq 0$, and 
$\operatorname{Supp}(B-\delta M)=\operatorname{Supp} B$. We have

$$
K_{X}+B-\delta M \equiv P+(1-\delta) M / Z
$$

By replacing the sequence of extremal rays with a subsequence, we can assume that each component $S$ of $M$ satisfies: either $S \cdot R_{i} \geq 0$ for every $i$, or $S \cdot R_{i}<0$ for every $i$. Pick a component $S$. If $S \cdot R_{i} \geq 0$ for each $i$, then by 3.3, we may assume that

$$
-3 \leq\left(K_{X}+B-\delta M\right) \cdot \Gamma_{i}<0
$$

and

$$
-3 \leq\left(K_{X}+B-\delta M-\tau S\right) \cdot \Gamma_{i}<0
$$

for every $i$ where $\tau>0$ is a small number. In particular, this means that $S \cdot \Gamma_{i}$ is bounded from below and above. On the other hand, if $S \cdot R_{i}<0$ for each $i$, then by considering $K_{X}+B-\delta M+\tau S$ and arguing similarly we can show that again $S \cdot \Gamma_{i}$ is bounded from below and above. In particular, there are only finitely many possibilities for the numbers $M \cdot \Gamma_{i}$. Therefore,

$$
\lim _{i \rightarrow \infty} P \cdot \Gamma_{i}=\lim _{i \rightarrow \infty}-\epsilon_{i} M \cdot \Gamma_{i}=0
$$

Write $K_{X}+B=\sum_{j} r_{j}\left(K_{X}+B_{j}\right)$ where $r_{j} \in(0,1], \sum r_{j}=1$, and $\left(X, B_{j}\right)$ are pairs with $B_{j}$ being rational. We can assume that each component of $B-B_{j}$ has irrational coefficient in $B$ hence $B-B_{j}$ and $M$ have no common components because $\operatorname{Supp} M \subseteq\lfloor B\rfloor$. Assume $\left(K_{X}+B_{j}\right) \cdot \Gamma_{i}<0$ for some $i, j$. Let $S$ be a component of $M$ such that $S \cdot \Gamma_{i}<0$, and let $S^{\nu}$ be its normalization. Let $K_{S^{\nu}}+B_{j, S^{\nu}}=\left.\left(K_{X}+B_{j}\right)\right|_{S^{\nu}}$ (see Section 4 for adjunction formulas of this type). On the other hand, by 3.3, there is an ample $\mathbb{Q}$-divisor $H$ such that $Q=K_{X}+B_{j}+H$ is nef and big and $R_{i}=Q^{\perp}$. Now the face $\left(\left.Q\right|_{S^{\nu}}\right)^{\perp}$ of $\overline{N E}\left(S^{\nu} / Z\right)$ is generated by finitely many curves $\Lambda_{1}^{\nu}, \ldots, \Lambda_{r}^{\nu}$ such that $\alpha_{j} \leq\left(K_{S^{\nu}}+B_{j, S^{\nu}}\right) \cdot \Lambda_{l}^{\nu}<0$ where $\alpha_{j}$ depends on $\left(S^{\nu}, B_{j, S^{\nu}}\right)$ but does not depend on $i$, by Tanaka [28, Theorem 4.4, Remark 4.5]. Let $\Lambda_{l}$ be the image of $\Lambda_{l}^{\nu}$ under the map $S^{\nu} \rightarrow X$. Since $R_{i}=Q^{\perp}$ and $Q \cdot \Lambda_{l}=0$, each $\Lambda_{l}$ also generates $R_{i}$. But as $\Gamma_{i}$ is extremal, perhaps after replacing the $\alpha_{j}$, we get

$$
\alpha_{j} \leq\left(K_{X}+B_{j}\right) \cdot \Lambda_{l} \leq\left(K_{X}+B_{j}\right) \cdot \Gamma_{i}<0
$$

by 3.1 .

On the other hand, since

$$
-3 \leq\left(K_{X}+B\right) \cdot \Gamma_{i}=\sum_{j} r_{j}\left(K_{X}+B_{j}\right) \cdot \Gamma_{i}<0
$$

for each $i$, we deduce that $\left(K_{X}+B_{j}\right) \cdot \Gamma_{i}$ is bounded from below and above for each $i, j$ which in turn implies that there are only finitely many possibilities for $\left(K_{X}+B\right) \cdot \Gamma_{i}$. Recalling that there are also finitely many possibilities for $M \cdot \Gamma_{i}$, we get a contradiction as

$$
0<P \cdot \Gamma_{i}=\left(K_{X}+B\right) \cdot \Gamma_{i}-M \cdot \Gamma_{i}
$$

but $\lim _{i \rightarrow \infty} P \cdot \Gamma_{i}=0$. 
3.7. LMMP using a weak Zariski decomposition. Let $(X, B)$ be a projective $\mathbb{Q}$-factorial pair of dimension 3 and $X \rightarrow Z$ a projective contraction such that $K_{X}+B \equiv P+M / Z$ where $P$ is nef $/ Z, M \geq 0$, and Supp $M \subseteq\lfloor B\rfloor$. Let $\mu$ be the largest number such that $P+\mu M$ is nef $/ Z$. Assume $\mu<1$. Then, by 3.6, there is an extremal ray $R / Z$ such that $\left(K_{X}+B\right) \cdot R<0$ and $(P+\mu M) \cdot R=0$. By replacing $P$ with $P+\mu M$ we may assume that $P \cdot R=0$. Assume that $R$ can be contracted by a projective morphism and that it gives a divisorial contraction or a $\log$ flip $X \rightarrow X^{\prime} / Z$ with $X^{\prime}$ being $\mathbb{Q}$-factorial. Obviously, $K_{X^{\prime}}+B^{\prime} \equiv P^{\prime}+M^{\prime} / Z$ where $P^{\prime}$ is nef $/ Z, M^{\prime} \geq 0$, and $\operatorname{Supp} M^{\prime} \subseteq\left\lfloor B^{\prime}\right\rfloor$. Continuing this process we obtain a particular kind of LMMP which we will refer to as the $L M M P$ using a weak Zariski decomposition or more specifically the $L M M P / Z$ on $K_{X}+B$ using $P+M$. When we need this LMMP below we will make sure that all the necessary ingredients exist.

\section{Adjunction}

The varieties in this section are over $k$ of arbitrary characteristic. We will use some of the results of Kollár [18] to prove an adjunction formula. Let $\Lambda$ be a DCC set of numbers in $[0,1]$. Then the hyperstandard set

$$
\mathfrak{S}_{\Lambda}=\left\{\frac{m-1}{m}+\sum \frac{l_{i} b_{i}}{m} \leq 1 \mid m \in \mathbb{N} \cup\{\infty\}, l_{i} \in \mathbb{Z}^{\geq 0}, b_{i} \in \Lambda\right\}
$$

also satisfies DCC.

Now let $(X, B)$ be a pair and $S$ a component of $\lfloor B\rfloor$. Let $S^{\nu} \rightarrow S$ be the normalization. Following a suggestion of Kollár, we will show that the pullback of $K_{X}+B$ to $S^{\nu}$ can be canonically written as $K_{S^{\nu}}+B_{S^{\nu}}$ for some $B_{S^{\nu}} \geq 0$ which is called the different. Moreover, if $(X, B)$ is lc outside a codimension 3 closed subset and if the coefficients of $B$ belong to $\Lambda$, then we show $B_{S^{\nu}}$ is a boundary with coefficients in $\mathfrak{S}_{\Lambda}$. When there is a log resolution $f: W \rightarrow X$, it is easy to define $B_{S^{\nu}}$ : let $K_{W}+B_{W}=f^{*}\left(K_{X}+B\right)$ and let $K_{T}+B_{T}=\left.\left(K_{W}+B_{W}\right)\right|_{T}$ where $T$ is the birational transform of $S$. Next, let $B_{S^{\nu}}$ be the pushdown of $B_{T}$ via $T \rightarrow S^{\nu}$. However, since existence of log resolutions is not known in general, we follow a different path, that is, that of [18, Section 4.1]. Actually, in this paper we will need this construction only when $\operatorname{dim} X \leq 3$ in which case $\log$ resolutions exist.

The characteristic 0 case of the results mentioned is due to Shokurov [26, Corollary 3.10]. His idea is to cut by appropriate hyperplane sections and reduce the problem to the case when $X$ is a surface. If the index of $K_{X}+S$ is 1 one proves the claim by direct calculations on a resolution. If the index is more than 1 one then uses the index 1 cover. Unfortunately this does not work in positive characteristic.

Proposition 4.1. Let $(X, B)$ be a pair, $S$ be a component of $\lfloor B\rfloor$, and $S^{\nu} \rightarrow S$ be the normalization. Then there is a canonically determined $\mathbb{R}$-divisor $B_{S^{\nu}} \geq 0$ such that

$$
K_{S^{\nu}}+\left.B_{S^{\nu}} \sim_{\mathbb{R}}\left(K_{X}+B\right)\right|_{S^{\nu}}
$$




\section{Existence of flips and minimal models for 3-folds in char $p \quad 17$}

where $\left.\right|_{S^{\nu}}$ means pullback to $S^{\nu}$ by the induced morphism $S^{\nu} \rightarrow X$.

Proof. If $K_{X}+B$ is $\mathbb{Q}$-Cartier, then the statement is proved in [18, 4.2 and 4.5]. In fact, $[18,4.2]$ defines $\Delta_{S^{\nu}}$ in general when $\Delta$ is a $\mathbb{Q}$-divisor with arbitrary rational coefficients, $S$ is a component of $\Delta$ with coefficient 1 , and $K_{X}+\Delta$ is $\mathbb{Q}$-Cartier (but $\Delta_{S^{\nu}}$ is not effective in general).

Let $U$ be the $\mathbb{R}$-vector space generated by the components of $B$. There is a rational affine subspace $V$ of $U$ containing $B$ and with minimal dimension. Since $V$ has minimal dimension, $\Delta-B$ is supported in the irrational part of $B$ for every $\Delta \in V$. Thus the coefficient of $S$ in $\Delta$ is 1 for every $\Delta \in V$.

Let $V_{\mathbb{Q}}$ be the underlying $\mathbb{Q}$-affine space of $V$. Let

$$
W_{\mathbb{Q}}=\left\{\Delta_{S^{\nu}} \mid \Delta \in V_{\mathbb{Q}}\right\}
$$

If $\Delta=\sum r_{j} \Delta^{j}$ where $r_{j}>0$ is rational, $\sum r_{j}=1$, and $\Delta^{j} \in V_{\mathbb{Q}}$, then the construction of $[18,4.2]$ shows that $\Delta_{S^{\nu}}=\sum r_{j} \Delta_{S^{\nu}}^{j}$. Therefore, $W_{\mathbb{Q}}$ is a $\mathbb{Q}$ affine space and the map $\alpha: V_{\mathbb{Q}} \rightarrow W_{\mathbb{Q}}$ sending $\Delta$ to $\Delta_{S^{\nu}}$ is an affine map. Letting $W$ be the $\mathbb{R}$-affine space generated by $W_{\mathbb{Q}}$, we get an induced affine map $V \rightarrow W$ which sends $B$ to some element $B_{S^{\nu}}$. Writing $B=\sum r_{j} \Delta^{j}$ where $r_{j}>0, \sum r_{j}=1$, and $0 \leq \Delta^{j} \in V_{\mathbb{Q}}$, we see that $B_{S^{\nu}}=\sum r_{j} \Delta_{S^{\nu}}^{j} \geq 0$. Moreover, by construction

$$
K_{S^{\nu}}+B_{S^{\nu}}=\left.\sum r_{j}\left(K_{S^{\nu}}+\Delta_{S^{\nu}}^{j}\right) \sim_{\mathbb{R}} \sum r_{j}\left(K_{X}+\Delta^{j}\right)\right|_{S^{\nu}}=\left.\left(K_{X}+B\right)\right|_{S^{\nu}}
$$

Note that in general $B_{S^{\nu}}$ is not a boundary, i.e. its coefficients may not be in $[0,1]$.

Proposition 4.2. Let $\Lambda \subseteq[0,1]$ be a DCC set of real numbers. Let $(X, B)$ be a pair, $S$ be a component of $\lfloor B\rfloor, S^{\nu} \rightarrow S$ be the normalization, and $B_{S^{\nu}}$ be the divisor given by Proposition 4.1. Assume that

- $(X, B)$ is lc outside a codimension 3 closed subset, and

- the coefficients of $B$ are in $\Lambda$.

Then $B_{S^{\nu}}$ is a boundary with coefficients in $\mathfrak{S}_{\Lambda}$. More precisely: write $B=$ $S+\sum_{i>2} b_{i} B_{i}$, let $V^{\nu}$ be a prime divisor on $S^{\nu}$ and let $V$ be its image on $S$; then there exists $m \in \mathbb{N} \cup\{\infty\}$ depending only on $X, S$ and $V$, and there exist nonnegative integers $l_{i}$ depending only on $X, S, B_{i}$ and $V$, such that the coefficient of $V^{\nu}$ in $B_{S^{\nu}}$ is equal to

$$
\frac{m-1}{m}+\sum_{i \geq 2} \frac{l_{i} b_{i}}{m}
$$

Lemma 4.3. Let $(X, B)$ be a pair which is lc outside a codimension 3 closed subset. Then we can write $B=\sum r_{j} B^{j}$ where $r_{j}>0, \sum r_{j}=1, B^{j}$ are $\mathbb{Q}$-boundaries, and $\left(X, B^{j}\right)$ are lc outside a codimension 3 closed subset. 
Proof. As in the proof of Proposition 4.1, there is a rational affine space $V$ of divisors, containing $B$, such that $K_{X}+\Delta$ is $\mathbb{R}$-Cartier for every $\Delta \in V$. The set of those $\Delta \in V$ with coefficients in $[0,1]$ is a rational polytope $\mathcal{P}$ containing $B$. We want to show that there is a rational polytope $\mathcal{L} \subseteq \mathcal{P}$, containing $B$, such that $(X, \Delta)$ is lc outside a fixed codimension 3 closed subset, for every $\Delta \in \mathcal{L}$. If $(X, B)$ has a $\log$ resolution, then existence of $\mathcal{L}$ can be proved using the same arguments as in $[26,1.3 .2]$.

The pair $(X, B)$ is log smooth outside some codimension 2 closed subset $Y$. In particular, $(X, \Delta)$ is lc outside $Y$, for every $\Delta \in \mathcal{P}$. Shrinking $X$ we can assume $Y$ is of pure codimension 2 and that $(X, B)$ is lc everywhere. Assume that for each component $R$ of $Y$, there is a rational polytope $\mathcal{L}_{R} \subseteq \mathcal{P}$, containing $B$, such that $(X, \Delta)$ is lc near the generic point of $R$, for every $\Delta \in \mathcal{L}_{R}$. Then we can take $\mathcal{L}$ to be any rational polytope, containing $B$, inside the intersection of the $\mathcal{L}_{R}$.

Existence of $\mathcal{L}_{R}$ is a local problem near the generic point of $R$. By replacing $X$ with Spec $\mathcal{O}_{X, R}$ we are reduced to the situation in which $X$ is a normal excellent scheme of dimension 2 (see $[18,3.3]$ for notion of lc pairs in this setting). Now $(X, B)$ has a log resolution (cf. see [23, page 28 and following remarks, and page 72$]$ ). So existence of $\mathcal{L}_{R}$ can be proved again as in $[26,1.3 .2]$.

Proof. (of Proposition 4.2) Assume that the proposition holds whenever $K_{X}+B$ is $\mathbb{Q}$-Cartier. In the general case, that is, when $K_{X}+B$ is only $\mathbb{R}$-Cartier, we can use Lemma 4.3 to write $B=\sum r_{j} B^{j}$ where $r_{j}>0, \sum r_{j}=1, B^{j}$ are $\mathbb{Q}$ boundaries, and $\left(X, B^{j}\right)$ are lc outside a codimension 3 closed subset. Moreover, we can assume $S$ is a component of $\left\lfloor B^{j}\right\rfloor$ for each $j$ since we can choose the $B^{j}$ so that $B-B^{j}$ are supported on the irrational part of $B$. Then $B_{S^{\nu}}=\sum r_{j} B_{S^{\nu}}^{j}$ (see the proof of Proposition 4.1). Write $B^{j}=S+\sum_{i \geq 2} b_{i}^{j} B_{i}$. By assumption, there exists $m \in \mathbb{N} \cup\{\infty\}$ depending only on $X, S$ and $V$, and there exist nonnegative integers $l_{i}$ depending only on $X, S, B_{i}$ and $V$, such that the coefficient of $V^{\nu}$ in $B_{S^{\nu}}^{j}$ is equal to

$$
\frac{m-1}{m}+\sum \frac{l_{i} b_{i}^{j}}{m}
$$

Therefore, the coefficient of $V^{\nu}$ in $B_{S^{\nu}}$ is equal to

$$
\frac{m-1}{m}+\sum_{j} r_{j}\left(\sum_{i} \frac{l_{i} b_{i}^{j}}{m}\right)=\frac{m-1}{m}+\sum_{i} l_{i}\left(\sum_{j} \frac{r_{j} b_{i}^{j}}{m}\right)=\frac{m-1}{m}+\sum_{i} \frac{l_{i} b_{i}}{m}
$$

So from now on we can assume that $K_{X}+B$ is $\mathbb{Q}$-Cartier. Determining the coefficient of $V^{\nu}$ in $B_{S^{\nu}}$ is a local problem near the generic point of $V$. As in the proof of Lemma 4.3, we can replace $X$ with $\operatorname{Spec} \mathcal{O}_{X, V}$ hence assume that $X$ is a normal excellent scheme of dimension 2, $S$ is one-dimensional, and $V$ is a closed point. Now $(X, B)$ is lc and the fact that $B_{S^{\nu}}$ is a boundary is proved in $[18,4.5]$.

Assume that $X$ is regular at $V$. If $S$ is not regular at $V$, then $B=S$ and the coefficient of $V^{\nu}$ in $B_{S^{\nu}}$ is equal to 1 (by [18, 3.45] or by blowing up $V$ 


\section{Existence of flips and minimal models for 3-folds in char $p \quad 19$}

and working on the blow up). But if $S$ is regular at $V$, then $S^{\nu} \rightarrow S$ is an isomorphism, $\left.\left(K_{X}+S\right)\right|_{S^{\nu}}=K_{S^{\nu}}, m=1$, and $B_{S^{\nu}}=\left.B\right|_{S^{\nu}}$. From these we can get the formula for the coefficient of $V^{\nu}$ as claimed. Thus we can assume $X$ is not regular at $V$.

Since $(X, B)$ is lc, $(X, S)$ is numerically lc (see [18, 3.3] for definition of numerical lc which is the same as lc except that $K_{X}+S$ may not be $\mathbb{Q}$-Cartier). If $(X, S)$ is not numerically plt, i.e. if there is an exceptional divisor over $V$ whose log discrepancy with respect to $(X, S)$ is 0 , then in fact $B=S$, and the coefficient of $V^{\nu}$ in $B_{S^{\nu}}$ is equal to 1 by $[18,3.45]$. Thus we can assume $(X, S)$ is numerically plt which in particular implies that $S$ is regular and that $S^{\nu} \rightarrow S$ is an isomorphism, by [18, 3.35].

Let $f: Y \rightarrow X$ be a $\log$ minimal resolution of $(X, S)$ as in $[18,2.25]$ and let $S^{\sim}$ be the birational transform of $S$. Then $S^{\sim} \rightarrow S$ is an isomorphism and the extended dual graph of the resolution is of the form

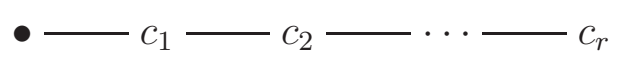

where $\bullet$ corresponds to $S^{\sim}, c_{i}=-E_{i}^{2}$, and $E_{1}, \ldots, E_{r}$ are the exceptional curves of $f$. Let $M=\left[-E_{i} \cdot E_{j}\right]$ be the minus of the intersection matrix of the resolution, and let $m=\operatorname{det} M$. Then by $[18,3.35 .1]$ we have

$$
K_{Y}+S^{\sim}+\sum e_{j} E_{j} \equiv 0 / X
$$

for certain $e_{j}>0$ and $e_{1}=\frac{m-1}{m}$.

Let $D \neq 0$ be an effective Weil divisor on $X$ with coefficients in $\mathbb{N}$. Let $d_{i}$ be the numbers so that $D^{\sim}+\sum d_{i} E_{i} \equiv 0 / X$ where $D^{\sim}$ is the birational transform of $D$. The $d_{i}$ satisfy the equations

$$
\left(\sum d_{j} E_{j}\right) \cdot E_{t}=-D^{\sim} \cdot E_{t}
$$

Since $M$ has integer entries and the numbers $-D^{\sim} \cdot E_{t}$ are integers, by Cramer's rule, we can write $d_{j}=\frac{n_{j}}{m}$ for certain $n_{j} \in \mathbb{N}$. Applying this to $D=B_{i}$, we have $B_{i}^{\sim}+\sum d_{i, j} E_{j} \equiv 0 / X$ for certain $d_{i, j}=\frac{n_{i, j}}{m}$ with $n_{i, j} \in \mathbb{N}$. But then

$$
K_{Y}+B^{\sim}+\sum e_{j}^{\prime} E_{j} \equiv 0 / X
$$

where $B^{\sim}$ is the birational transform of $B$ and $e_{j}^{\prime}=e_{j}+\sum_{i \geq 2} \frac{n_{i, j} b_{i}}{m}$. In particular, $e_{1}^{\prime}=\frac{m-1}{m}+\sum_{i \geq 2} \frac{l_{i} b_{i}}{m}$ where we put $l_{i}:=n_{i, 1}$. Now the coefficient of $V^{\nu}$ in $B_{S^{\nu}}$ is simply the coefficient of the divisor $\left.e_{1}^{\prime} E_{1}\right|_{S^{\sim}}$ which is nothing but $e_{1}^{\prime}$.

\section{SPECIAL TERMinAtion}

All the varieties and algebraic spaces in this section are over $k$ of char $p>0$ unless stated otherwise. 


\subsection{Reduced components of boundaries of dlt pairs.}

Lemma 5.2. Let $(X, B)$ be an lc pair of dimension 3 and $S$ a component of $\lfloor B\rfloor$. Then we have:

(1) if the coefficients of $B$ are standard, then the coefficients of $B_{S^{\nu}}$ are also standard;

(2) if $k$ has char $p>5$ and $(X, B)$ is $\mathbb{Q}$-factorial dlt, then $S$ is normal.

Proof. (1) This follows from Kollár [18, Corollary 3.45] (see also [18, 4.4]).

(2) We may assume $B=S$ by discarding all the other components, in particular, $(X, B)$ is plt hence $\left(S^{\nu}, B_{S^{\nu}}\right)$ is klt. By $(1), B_{S^{\nu}}$ has standard coefficients. By [13, Theorem 3.1], $\left(S^{\nu}, B_{S^{\nu}}\right)$ is actually strongly $F$-regular. Therefore, $S$ is normal by $[13$, Theorem 4.1].

5.3. Pl-extremal rays. Let $(X, B)$ be a projective $\mathbb{Q}$-factorial dlt pair of dimension 3. A $K_{X}+B$-negative extremal ray $R$ is called a pl-extremal ray if $S \cdot R<0$ for some component $S$ of $\lfloor B\rfloor$. This is named after Shokurov's pl-flips.

Assume that $k$ has char $p>5$. Now as in 3.3, assume that $\mathcal{C}=\overline{N E}(X / Z)$ for some projective contraction $X \rightarrow Z$ such that $K_{X}+B \equiv P+M / Z$ where $P$ is nef $/ Z$ and $M \geq 0$, or $\mathcal{C}=N^{\perp}$ for some nef and big $\mathbb{Q}$-divisor $N$. Let $R$ be a $K_{X}+B$-negative pl-extremal ray of $\mathcal{C}$. By 3.3 , we can find a $\mathbb{Q}$-boundary $\Delta$ and an ample $\mathbb{Q}$-divisor $H$ such that $\lfloor\Delta\rfloor=S,\left(K_{X}+\Delta\right) \cdot R<0$ and $L=K_{X}+\Delta+H$ is nef and big with $L^{\perp}=R$. Let $X \rightarrow V$ be the contraction associated to $L$ which contracts $R$ to an algebraic space. Every curve contracted by $X \rightarrow V$ is inside $S$. So the exceptional locus $\mathbb{E}(L)$ of $L$ is inside $S$. Thus $L$ is semiample by 2.13. Therefore $X \rightarrow V$ is a projective contraction. In other words, pl-extremal rays can be contracted by projective morphisms. This was proved in [13, Theorem 5.4] when $K_{X}+B$ is pseudo-effective. The extremal rays that appear below are often pl-extremal rays.

If $X \rightarrow V$ is a divisorial contraction put $X^{\prime}=V$ but if it is a flipping contraction assume $X \rightarrow X^{\prime} / V$ is its flip. Then it is not hard to see that in any case $X^{\prime}$ is $\mathbb{Q}$-factorial, by the following argument [30]: we treat the divisorial case; the flipping case can be proved similarly. We can assume that $B$ is a $\mathbb{Q}$-boundary and $\Delta=B$. Let $D^{\prime}$ be a prime divisor on $X^{\prime}$ and $D$ its birational transform on $X$. There are rational numbers $\epsilon>0$ and $\delta$ such that $M:=K_{X}+B+H+\epsilon D+\delta S$ is nef and big, $M \equiv 0 / V, H+\epsilon D+\delta S$ is ample, and $\mathbb{E}(M)=\mathbb{E}(L)=S$. Since $\left.M\right|_{S}$ is semi-ample, $M$ is semi-ample by Theorem 2.9. That is, $M$ is the pullback of some ample divisor $M^{\prime}$ on $X^{\prime}$. But then $\epsilon D^{\prime}=M^{\prime}-L^{\prime}$ is $\mathbb{Q}$-Cartier hence $D^{\prime}$ is $\mathbb{Q}$-Cartier.

5.4. Special termination. The following important result is proved just like in characteristic 0 . We include the proof for convenience.

Proposition 5.5. Let $(X, B)$ be a projective $\mathbb{Q}$-factorial dlt pair of dimension 3 over $k$ of char $p>5$. Assume that we are given an LMMP on $K_{X}+B$, say $X_{i} \rightarrow X_{i+1} / Z_{i}$ where $X_{1}=X$ and each $X_{i} \rightarrow X_{i+1} / Z_{i}$ is a flip, or a divisorial 


\section{Existence of flips and minimal models for 3-folds in char $p \quad 21$}

contraction with $X_{i+1}=Z_{i}$. Then after finitely many steps, each remaining step of the LMMP is an isomorphism near the lc centres of $(X, B)$.

Proof. There are only finitely many lc centres and no new one can be created in the process, so we may assume that the LMMP does not contract any lc centre. In particular, we can assume that the LMMP is an isomorphism near each lc centre of dimension zero.

Now let $C$ be an lc centre of dimension one. Since $(X, B)$ is dlt, $C$ is a component of the intersection of two components $S, S^{\prime}$ of $\lfloor B\rfloor$. Let $C_{i}, S_{i} \subset X_{i}$ be the birational transforms of $C, S$. Applying Lemma 5.2, we can see that $C_{i}, S_{i}$ are normal. By adjunction, we can write $\left.\left(K_{X_{i}}+B_{i}\right)\right|_{S_{i}}=K_{S_{i}}+B_{S_{i}}$ where the coefficient of $C_{i}$ in $B_{S_{i}}$ is one. Applying adjunction once more, we can write the pullback of $K_{S_{i}}+B_{S_{i}}$ to $C_{i}$ as $K_{C_{i}}+B_{C_{i}}$ for some boundary $B_{C_{i}}$. Since $C_{i} \simeq C_{i+1}$, we will use the notation $\left(C, B_{i, C}\right)$ instead of $\left(C_{i}, B_{C_{i}}\right)$. Since each step of the LMMP makes the divisor $K_{X}+B$ "smaller",

$$
K_{C}+B_{i, C} \geq K_{C}+B_{i+1, C}
$$

hence $B_{i, C} \geq B_{i+1, C}$ for every $i$. By Propositions 4.2 , the coefficients of $B_{S_{i}}$ and $B_{i, C}$ belong to some fixed DCC set. Therefore $B_{i, C}=B_{i+1, C}$ for every $i \gg 0$ which implies that after finitely many steps, each remaining step of the LMMP is an isomorphism near $C_{i}$.

From now on we may assume that all the steps of the LMMP are flips. Let $S$ be any lc centre of dimension 2, i.e. a component of $B$ with coefficient one. If $S_{i}$ intersects the exceptional locus $E_{i}$ of $X_{i} \rightarrow Z_{i}$, then no other component of $\left\lfloor B_{i}\right\rfloor$ can intersect the exceptional locus: assume that another component $T_{i}$ intersects the exceptional locus; if either $S_{i}$ or $T_{i}$ contains $E_{i}$, then $S_{i} \cap$ $T_{i}$ intersects $E_{i}$; but $S_{i} \cap T_{i}$ is a union of lc centres of dimension one and this contradicts the last paragraph; so none of $S_{i}, T_{i}$ contains $E_{i}$. But then both contain the exceptional locus of $X_{i+1} \rightarrow Z_{i}$ and similar arguments give a contradiction.

Assume $D_{i} \subset S_{i}$ is a component of the exceptional locus of $X_{i} \rightarrow Z_{i-1}$ where $i>1$. Then the log discrepancy of $D_{i}$ with respect to $\left(S_{1}, B_{S_{1}}\right)$ is less than one. Moreover, we can assume that the generic point of the centre of $D_{i}$ on $S_{1}$ is inside the klt locus of $\left(S_{1}, B_{S_{1}}\right)$ by the last paragraph. But there can be at most finitely many such $D_{i}$ (as prime divisors on birational models of $S_{1}$ ). Since the coefficients of $D_{i}$ in $B_{S_{i}}$ belongs to a DCC set, the coefficient of $D_{i}$ stabilizes. Therefore after finitely many steps, $S_{i}$ cannot contain any component of the exceptional locus of $X_{i} \rightarrow Z_{i-1}$. So we get a sequence $S_{i}-\rightarrow S_{i+1}$ of birational morphisms which are isomorphisms if $i \gg 0$. In particular, $S_{i}$ is disjoint from $E_{i}$ for $i \gg 0$.

\section{EXISTENCE OF LOG FLIPS}

In this section, we first prove that generalized flips exist (6.3). Next we prove Theorem 1.1 in the projective case, that is, when $X$ is projective. The general case of Theorem 1.1 is proved in Section 8 where $X$ is quasi-projective. 
6.1. Divisorial and flipping extremal rays. Let $(X, B)$ be a projective $\mathbb{Q}$ factorial pair of dimension 3 over $k$ of char $p>0$, and let $R$ be a $K_{X}+B$ negative extremal ray. Assume that there is a nef and big $\mathbb{Q}$-divisor $L$ such that $R=L^{\perp}$. We say $R$ is a divisorial extremal ray if $\operatorname{dim} \mathbb{E}(L)=2$. But we say $R$ is a flipping extremal ray if $\operatorname{dim} \mathbb{E}(L)=1$. By 3.3, such rays can be contracted to algebraic spaces. By 3.2, when $K_{X}+B$ is pseudo-effective, each $K_{X}+B$-negative extremal ray is either a divisorial extremal ray or a flipping extremal ray. We will show below (1.5) that any divisorial or flipping extremal ray can actually be contracted by a projective morphism if $(X, B)$ is dlt and $p>5$. However, we still need contractions to algebraic spaces as an auxiliary tool.

6.2. Existence of generalized flips. We recall the definition of generalized flips which was introduced in [13]. Let $(X, B)$ be a projective $\mathbb{Q}$-factorial pair of dimension 3 over $k$ char $p>0$, and let $R$ be a $K_{X}+B$-negative flipping extremal ray. We say that the generalized flip of $R$ exists (see [13, after Theorem 5.6]) if there is a birational map $X \rightarrow X^{+} / V$ which is an isomorphism in codimension one, $X^{+}$is $\mathbb{Q}$-factorial projective, and $K_{X^{+}}+B^{+}$is numerically positive on any curve contracted by $X^{+} \rightarrow V$.

Theorem 6.3. Let $(X, B)$ be a projective $\mathbb{Q}$-factorial dlt pair of dimension 3 over $k$ of char $p>5$. Let $R$ be a $K_{X}+B$-negative flipping extremal ray. Then the generalized flip of $R$ exists.

The theorem was proved in [13, Theorem 5.6] when $B$ has standard coefficients and $K_{X}+B$ is pseudo-effective.

Proof. This proof (as well as the proof of [13, Theorem 5.6]) is modeled on the proof of Shokurov's reduction theorem [25, Theorem 1.2]. Since $R$ is a flipping extremal ray, by definition, there is a nef and big $\mathbb{Q}$-divisor $L$ such that $R=L^{\perp}$. Moreover, $L$ is endowed with a map $X \rightarrow V$ to an algebraic space which contracts the curves generating $R$. Note that if $B^{\prime}$ is another boundary such that $\left(K_{X}+B^{\prime}\right) \cdot R<0$, then the generalized flip exists for $(X, B)$ if and only if it exists for $\left(X, B^{\prime}\right)$. This follows from the fact that $K_{X}+B \equiv t\left(K_{X}+B^{\prime}\right) / V$ for some number $t>0$ where the numerical equivalence means that $K_{X}+B-t\left(K_{X}+B^{\prime}\right)$ is numerically trivial on any curve contracted by $X \rightarrow V$.

Let $\mathfrak{S}$ be the set of standard coefficients as defined in the introduction. Define

$$
\zeta(X, B)=\#\{S \mid S \text { is a component of } B \text { and its coefficient is not in } \mathfrak{S}\}
$$

Assume that the generalized flip of $R$ does not exist. We will derive a contradiction. We can assume that $\zeta(X, B)$ is minimal, that is, we may assume that generalized flips always exist for pairs with smaller $\zeta$. We can decrease the coefficients of $\lfloor B\rfloor$ slightly so that $(X, B)$ becomes klt and $\zeta(X, B)$ is unchanged. In addition, each component $S$ of $B$ whose coefficient is not in $\mathfrak{S}$ satisfies $S \cdot R<0$ otherwise we can discard $S$ and decrease $\zeta(X, B)$ which is not possible by the minimality assumption. 


\section{Existence of flips and minimal models for 3-folds in char $p$}

23

First assume that $\zeta(X, B)>0$. Choose a component $S$ of $B$ whose coefficient $b$ is not in $\mathfrak{S}$. There is a positive number $a$ such that $K_{X}+B \equiv a S / V$. Let $g: W \rightarrow X$ be a log resolution, and let $B_{W}=B^{\sim}+E$ and $\Delta_{W}=B_{W}+(1-$ b) $S^{\sim}$ where $E$ is the reduced exceptional divisor of $g$ and $B^{\sim}, S^{\sim}$ are birational transforms. Note that $\left\lfloor B_{W}\right\rfloor=E$ and $\left\lfloor\Delta_{W}\right\rfloor=S^{\sim}+E$. Since $(X, B)$ is klt,

$$
K_{W}+\Delta_{W}=K_{W}+B_{W}+(1-b) S^{\sim}=g^{*}\left(K_{X}+B\right)+G+(1-b) S^{\sim}
$$

where $G$ is effective and its support is equal to the support of $E$. Thus

$$
K_{W}+\Delta_{W} \equiv g^{*}(a S)+G+(1-b) S^{\sim}=(a+1-b) S^{\sim}+F / V
$$

where $F$ is effective and $\operatorname{Supp} F=\operatorname{Supp} E$. By construction, we have

$$
\operatorname{Supp}\left(S^{\sim}+F\right)=\left\lfloor\Delta_{W}\right\rfloor \text { and } \zeta\left(W, \Delta_{W}\right)<\zeta(X, B)
$$

Run an LMMP $/ V$ on $K_{W}+\Delta_{W}$ with scaling of some ample divisor, as in 3.5. Recall that this is an LMMP/C on $K_{W}+\Delta_{W}$ where $\mathcal{C}=N^{\perp}$ and $N$ is the pullback of the nef and big $\mathbb{Q}$-divisor $L$. In each step some component of $\left\lfloor\Delta_{W}\right\rfloor$ is negative on the corresponding extremal ray. So such extremal rays are pl-extremal rays, they can be contracted by projective morphisms, and the $\mathbb{Q}$-factorial property is preserved (see 5.3). Moreover, if we encounter a flipping contraction, then its generalized flip exists because $\zeta\left(W, \Delta_{W}\right)<\zeta(X, B)$ and because we chose $\zeta(X, B)$ to be minimal; the flip is a usual one since its extremal ray is contracted projectively. By special termination (5.5), the LMMP terminates on some model $Y / V$.

Now run an LMMP $/ V$ on $K_{Y}+B_{Y}$ with scaling of $(1-b) S_{Y}$ where $B_{Y}$ is the pushdown of $B_{W}$ and $S_{Y}$ is the pushdown of $S^{\sim}$. Since we have the numerical equivalence $K_{Y}+B_{Y} \equiv a S_{Y}+F_{Y} / V$ and Supp $F_{Y}=\left\lfloor B_{Y}\right\rfloor$, in each step of the LMMP the corresponding extremal ray intersects some component of $\left\lfloor B_{Y}\right\rfloor$ negatively hence they are pl-extremal rays and they can be contracted by projective morphisms (5.3). Moreover, if one of these rays gives a flipping contraction, then its generalized flip exists because $K_{Y}+B_{Y}-b S_{Y}$ is negative on that ray and $\zeta\left(Y, B_{Y}-b S_{Y}\right)<\zeta(X, B)$. Note that again such flips are usual flips. The LMMP terminates on a model $X^{+}$by special termination.

Let $h: W^{\prime} \rightarrow X$ and $e: W^{\prime} \rightarrow X^{+}$be a common resolution. Now the negativity lemma (2.3) applied to the divisor $h^{*}\left(K_{X}+B\right)-e^{*}\left(K_{X^{+}}+B^{+}\right)$over $X$ implies that

$$
h^{*}\left(K_{X}+B\right)-e^{*}\left(K_{X^{+}}+B^{+}\right) \geq 0
$$

Thus every component $D$ of $E$ is contracted over $X^{+}$because

$$
0<a(D, X, B) \leq a\left(D, X^{+}, B^{+}\right)
$$

Therefore $X \rightarrow X^{+}$is an isomorphism in codimension one. It is enough to show that $K_{X^{+}}+B^{+}$is numerically positive $/ V$. Let $H^{+}$be an ample divisor on $X^{+}$and $H$ its birational transform on $X$. There is a positive number $c$ such that $K_{X}+B \equiv c H / V$ hence $K_{X^{+}}+B^{+} \equiv c H^{+} / V$ which implies that $K_{X^{+}}+B^{+}$ is numerically positive $/ V$. So we have constructed the generalized flip and this contradicts our assumptions above. 
Now assume that $\zeta(X, B)=0$. If $K_{X}+B$ is pseudo-effective, then we can simply apply [13, Theorem 5.6] to get a contradiction. Unfortunately, $K_{X}+B$ may not be pseudo-effective (note that even if we originally start with a pseudoeffective log divisor we may end up with a non-pseudo-effective $K_{X}+B$ since we decreased some coefficients). However, this is not a problem because the proof of [13, Theorem 5.6] still works. Since there is a nef and big $\mathbb{Q}$-divisor $L$ with $L \cdot R=0$, there is a prime divisor $S$ with $S \cdot R<0$. There is a number $a>0$ such that $K_{X}+B \equiv a S / V$. Now take a log resolution $g: W \rightarrow X$ and define $B_{W}$ and $\Delta_{W}$ as above (if $S$ is not a component of $B$ simply let $b=0$ ). Run an LMMP $/ V$ on $K_{W}+\Delta_{W}$. The extremal rays in the process are all pl-extremal rays hence they can be contracted by projective morphisms. Moreover, if we encounter a flipping contraction, then its flip exists by [13, Theorem 4.12] because all the coefficients of $\Delta_{W}$ are standard. The LMMP terminates on some model $Y$ by the special termination. Next, run the LMMP $/ V$ on $K_{Y}+B_{Y}$ with scaling of $(1-b) S_{Y}$. Again, the extremal rays in the process are all pl-extremal rays hence they can be contracted by projective morphisms. Moreover, if we encounter a flipping contraction, then its flip exists by [13, Theorem 4.12] because all the coefficients of $B_{Y}$ are standard. The LMMP terminates on some model $X^{+}$by the special termination. The rest of the argument goes as before.

\subsection{Proof of $\mathbf{1 . 1}$ in the projective case.}

Proof. (of Theorem 1.1 in the projective case) Assume that $X$ is projective. Then by Theorem 6.3, the generalized flip of the extremal ray of $X \rightarrow Z$ exists. But since $X \rightarrow Z$ is a projective contraction, the generalized flip is a usual flip.

If $X$ is only quasi-projective, we postpone the proof to Section 8. Until then we need flips only in the projective case.

\section{Crepant models}

7.1. Divisorial extremal rays. The next lemma is essentially [13, Theorem $5.6(2)]$.

Lemma 7.2. Let $(X, B)$ be a projective $\mathbb{Q}$-factorial dlt pair of dimension 3 over $k$ of char $p>5$. Let $R$ be a $K_{X}+B$-negative divisorial extremal ray. Then $R$ can be contracted by a projective morphism $X \rightarrow Z$ where $Z$ is $\mathbb{Q}$-factorial.

Proof. We may assume that $(X, B)$ is klt. Since $R$ is a divisorial extremal ray, by definition, there is a nef and big $\mathbb{Q}$-divisor $L$ such that $R=L^{\perp}$ and $\operatorname{dim} \mathbb{E}(L)=2$. Moreover, $R$ can be contracted by a map $X \rightarrow V$ to an algebraic space. There is a prime divisor $S$ with $S \cdot R<0$. In particular, $\mathbb{E}(L) \subseteq S$ and $S$ is the only prime divisor contracted by $X \rightarrow V$. There is a number $a>0$ such that $K_{X}+B \equiv a S / V$. Let $g: W \rightarrow X$ be a log resolution and define $\Delta_{W}$ as in the proof of Theorem 6.3. Run an LMMP/V on $K_{W}+\Delta_{W}$. As in 6.3, the extremal rays in the process are pl-extremal rays hence they are contracted projectively and the LMMP terminates with a model $Z$. We are done if we 
Existence of flips and minimal models for 3-folds in char $p \quad 25$

show that $Z \rightarrow V$ is an isomorphism (the $\mathbb{Q}$-factoriality claim follows from 5.3). Assume this is not the case.

Recall that

$$
K_{W}+\Delta_{W} \equiv(a+1-b) S^{\sim}+F / V
$$

and now $(a+1-b) S^{\sim}+F$ is exceptional/ $V$. In particular, $(a+1-b) S_{Z}+F_{Z}$ is effective, exceptional and nef $/ V$.

Let $H_{Z}$ be a general ample divisor on $Z$ and $H$ its birational transform on $X$. There is a number $t \geq 0$ such that $H+t S \equiv 0 / V$. Therefore there is an effective and exceptional $/ V$ divisor $P_{Z}$ such that $H_{Z}+P_{Z} \equiv 0 / V$. Note that $\operatorname{Supp} P_{Z}$ contains all the exceptional divisors of $Z \rightarrow V$ hence $\operatorname{Supp} P_{Z}=\operatorname{Supp} F_{Z}$. Moreover, $P_{Z} \neq 0$ otherwise $H_{Z} \equiv 0 / V$ hence $Z \rightarrow V$ is an isomorphism which is not the case by assumption. This also shows that $F_{Z} \neq 0$.

Let $s$ be the smallest number such that

$$
Q_{Z}:=(a+1-b) S_{Z}+F_{Z}-s P_{Z} \leq 0
$$

Then $Q_{Z}$ is numerically positive over $V$ and there is some prime exceptional $/ V$ divisor $D$ which is not a component of $Q_{Z}$. This is not possible since $Q_{Z}$ cannot be numerically positive on the general curves of $D$ contracted $/ V$.

\subsection{Projectivization and dlt models.}

Lemma 7.4. Let $X$ be a normal projective variety over $k$ and $D \neq X$ a closed subset. Then there is a reduced effective Cartier divisor $H$ whose support contains $D$.

Proof. We may assume that each irreducible component of $D$ is a prime divisor hence we can think of $D$ as a reduced Weil divisor. Let $A$ be a sufficiently ample divisor. Let $U$ be the smooth locus of $X$. Since $\left.(A-D)\right|_{U}$ is sufficiently ample, we can choose a reduced effective divisor $H^{\prime}$ with no common components with $D$ such that $\left.\left.H^{\prime}\right|_{U} \sim(A-D)\right|_{U}$. This extends to $X$ and gives $H^{\prime} \sim A-D$. Now $H:=H^{\prime}+D \sim A$ is Cartier and satisfies the requirements.

The next few results are standard consequences of special termination (cf. [3, Lemma 3.3][13, Theorem 6.1]).

Lemma 7.5. Let $(X, B)$ be an lc pair of dimension 3 over $k$ of char $p>5$, and let $\bar{X}$ be a projectivization of $X$. Then there is a projective $\mathbb{Q}$-factorial dlt pair $\left(\bar{Y}, B_{\bar{Y}}\right)$ with a birational morphism $\bar{Y} \rightarrow \bar{X}$ satisfying the following:

- $K_{\bar{Y}}+B_{\bar{Y}}$ is nef/ $\bar{X}$,

- let $Y$ be the inverse image of $X$ and $B_{Y}=\left.B_{\bar{Y}}\right|_{Y}$; then $\left(Y, B_{Y}\right)$ is a $\mathbb{Q}$ factorial dlt model of $(X, B)$.

Proof. We may assume that $\bar{X}$ is normal. By Lemma 7.4, there is a reduced effective Cartier divisor $H$ containing the complement of $X$ in $\bar{X}$. We may assume that $H$ has no common components with $B$. Let $f: \bar{W} \rightarrow \bar{X}$ be a log resolution. Now let $B_{\bar{W}}$ be the sum of the reduced exceptional divisor of $f$ and 
the birational transform of $B$, and let $\Delta_{\bar{W}}$ be the sum of $B_{\bar{W}}$ and the birational transform of $H$.

Run the LMMP $/ \bar{X}$ on $K_{\bar{W}}+\Delta_{\bar{W}}$ inductively as follows. Assume that we have arrived at a model $\bar{Y}$. Let $R$ be a $K_{\bar{Y}}+\Delta_{\bar{Y}}$-negative extremal ray $/ \bar{X}$. Let $\bar{Y} \rightarrow \bar{Z}$ be the contraction of $R$ to an algebraic space, and let $L$ be a nef and big $\mathbb{Q}$-divisor with $L^{\perp}=R$. Any curve contracted by $\bar{Y} \rightarrow \bar{Z}$ is also contracted over $\bar{X}$. If $\operatorname{dim} \mathbb{E}(L)=2$, then $R$ is a divisorial extremal ray hence $\bar{Y} \rightarrow \bar{Z}$ is a projective contraction by Lemma 7.2. In this case, we continue the program with $\bar{Z}$. Now assume that $\operatorname{dim} \mathbb{E}(L)=1$. Let $C$ be a connected component of $\mathbb{E}(L)$ and $P$ its image in $\bar{X}$ which is just a point. If $P \in \operatorname{Supp} H$, then $C$ is contained in some component of the pullback of $H$ hence it is contained in some component of $\left\lfloor\Delta_{\bar{Y}}\right\rfloor$. In this case, $\bar{Y} \rightarrow \bar{Z}$ is again a projective contraction by 2.13. Now assume that $P$ does not belong to the support of $H$. Since $(X, B)$ is lc, over $X \backslash H$ the divisor

$$
K_{\bar{W}}+\Delta_{\bar{W}}-f^{*}\left(K_{X}+B\right)
$$

is effective and exceptional $/ \bar{X}$ hence some component of $\Delta_{\bar{Y}}$ intersects $R$ negatively which implies again that the contraction $\bar{Y} \rightarrow \bar{Z}$ is projective. Therefore in any case $R$ can be contracted by a projective morphism and we can continue the LMMP as usual. The required flips exist by the results of Section 6. By special termination (5.5), the LMMP terminates say on $\bar{Y}$.

Next, we run the LMMP $/ \bar{X}$ on $K_{\bar{Y}}+B_{\bar{Y}}$ with scaling of $\Delta_{\bar{Y}}-B_{\bar{Y}}$ as in 3.5. Note that $\Delta_{\bar{Y}}-B_{\bar{Y}}$ is nothing but the birational transform of $H$. Since the pullback of $H$ is numerically trivial over $\bar{X}$, each extremal ray in the process intersects some exceptional divisor negatively hence such extremal rays can be contracted by projective morphisms. Moreover, the required flips exist and by special termination the LMMP terminates on a model which we may again denote by $\bar{Y}$. Now let $Y$ be the inverse image of $X$ under $g: \bar{Y} \rightarrow \bar{X}$ and let $B_{Y}$ be the restriction of $B_{\bar{Y}}$ to $Y$. Then $\left(Y, B_{Y}\right)$ is a $\mathbb{Q}$-factorial dlt model of $(X, B)$ because $K_{Y}+B_{Y}-g^{*}\left(K_{X}+B\right)$ is effective and exceptional hence zero as it is nef/ $X$.

Proof. (of Theorem 1.6) This is already proved in Lemma 7.5.

\subsection{Extraction of divisors and terminal models.}

Lemma 7.7. Let $(X, B)$ be an lc pair of dimension 3 over $k$ of char $p>5$ and let $\left\{D_{i}\right\}_{i \in I}$ be a finite set of exceptional/X prime divisors (on birational models of $X)$ such that $a\left(D_{i}, X, B\right) \leq 1$. Then there is a $\mathbb{Q}$-factorial dlt pair $\left(Y, B_{Y}\right)$ with a projective birational morphism $Y \rightarrow X$ such that

- $K_{Y}+B_{Y}$ is the crepant pullback of $K_{X}+B$,

- every exceptional/X prime divisor $E$ of $Y$ is one of the $D_{i}$ or $a(E, X, B)=0$,

- the set of exceptional/X prime divisors of $Y$ includes $\left\{D_{i}\right\}_{i \in I}$. 
Proof. By Lemma 7.5, we can assume that $(X, B)$ is projective $\mathbb{Q}$-factorial dlt. Let $f: W \rightarrow X$ be a $\log$ resolution and let $\left\{E_{j}\right\}_{j \in J}$ be the set of prime exceptional divisors of $f$. We can assume that for some $J^{\prime} \subseteq J,\left\{E_{j}\right\}_{j \in J^{\prime}}=\left\{D_{i}\right\}_{i \in I}$. Now define

$$
K_{W}+B_{W}:=f^{*}\left(K_{X}+B\right)+\sum_{j \notin J^{\prime}} a\left(E_{j}, X, B\right) E_{j}
$$

which ensures that if $j \notin J^{\prime}$, then $E_{j}$ is a component of $\left\lfloor B_{W}\right\rfloor$. Run an LMMP $/ X$ on $K_{W}+B_{W}$ which would be an LMMP on $\sum_{j \notin J^{\prime}} a\left(E_{j}, X, B\right) E_{j}$. So each extremal ray in the process intersects some component of $\left\lfloor B_{W}\right\rfloor$ negatively hence such rays can be contracted by projective morphisms (5.3), the required flips exists (Section 6), and the LMMP terminates by special termination (5.5), say on a model $Y$. Now $\left(Y, B_{Y}\right)$ satisfies all the requirements.

Proof. (of Corollary 1.7) Apply Lemma 7.7 by taking $\left\{D_{i}\right\}_{i \in I}$ to be the set of all prime divisors with $\log$ discrepancy $a\left(D_{i}, X, B\right) \leq 1$.

\section{EXISTENCE OF LOG Minimal MODELS}

8.1. Weak Zariski decompositions. Let $D$ be an $\mathbb{R}$-Cartier divisor on a normal variety $X$ and $X \rightarrow Z$ a projective contraction over $k$. A weak Zariski decomposition/ $Z$ for $D$ consists of a projective birational morphism $f: W \rightarrow X$ from a normal variety, and a numerical equivalence $f^{*} D \equiv P+M / Z$ such that

(1) $P$ and $M$ are $\mathbb{R}$-Cartier divisors,

(2) $P$ is nef $/ Z$, and $M \geq 0$.

We then define $\theta(X, B, M)$ to be the number of those components of $f_{*} M$ which are not components of $\lfloor B\rfloor$.

8.2. From weak Zariski decompositions to minimal models. We use the methods of [2], which is somewhat similar to [5, §5], to prove the following result.

Proposition 8.3. Let $(X, B)$ be a projective lc pair of dimension 3 over $k$ of char $p>5$, and $X \rightarrow Z$ a projective contraction. Assume that $K_{X}+B$ has a weak Zariski decomposition $/ Z$. Then $(X, B)$ has a log minimal model over $Z$.

Proof. Assume that $\mathfrak{W}$ is the set of pairs $(X, B)$ and projective contractions $X \rightarrow Z$ such that

L: $(X, B)$ is projective, lc of dimension 3 over $k$,

Z: $K_{X}+B$ has a weak Zariski decomposition $/ Z$, and

$\mathbf{N}:(X, B)$ has no $\log$ minimal model over $Z$.

Clearly, it is enough to show that $\mathfrak{W}$ is empty. Assume otherwise and let $(X, B)$ and $X \rightarrow Z$ be in $\mathfrak{W}$. Let $f: W \rightarrow X, P$ and $M$ be the data given by a weak Zariski decomposition $/ Z$ for $K_{X}+B$ as in 8.1. Assume in addition that 
$\theta(X, B, M)$ is minimal. Perhaps after replacing $f$ we can assume that $f$ gives a $\log$ resolution of $\left(X, \operatorname{Supp}\left(B+f_{*} M\right)\right)$. Let $B_{W}=B^{\sim}+E$ where $B^{\sim}$ is the birational transform of $B$ and $E$ is the reduced exceptional divisor of $f$. Then

$$
K_{W}+B_{W}=f^{*}\left(K_{X}+B\right)+F \equiv P+M+F / Z
$$

is a weak Zariski decomposition where $F \geq 0$ is exceptional/ $X$. Moreover,

$$
\theta\left(W, B_{W}, M+F\right)=\theta(X, B, M)
$$

and any log minimal model of $\left(W, B_{W}\right)$ is also a log minimal model of $(X, B)$ $\left[2\right.$, Remark 2.4]. So by replacing $(X, B)$ with $\left(W, B_{W}\right)$ and $M$ with $M+F$ we may assume that $W=X,(X, \operatorname{Supp}(B+M))$ is $\log$ smooth, and that $K_{X}+B \equiv P+M / Z$.

First assume that $\theta(X, B, M)=0$, that is, Supp $M \subseteq\lfloor B\rfloor$. Run the LMMP $/ Z$ on $K_{X}+B$ using $P+M$ as in 3.7. Obviously, $M$ negatively intersects each extremal ray in the process, and since Supp $M \subseteq\lfloor B\rfloor$, the rays are pl-extremal rays. Therefore those rays can be contracted by projective morphisms (5.3), the required flips exist (Section 6), and the LMMP terminates by special termination (5.4). Thus we get a $\log$ minimal model of $(X, B)$ over $Z$ which contradicts the assumption that $(X, B)$ and $X \rightarrow Z$ belong to $\mathfrak{W}$. For the rest of the proof we do not use LMMP.

From now on we assume that $\theta(X, B, M)>0$. Define

$$
\alpha:=\min \left\{t>0 \mid\left\lfloor(B+t M)^{\leq 1}\right\rfloor \neq\lfloor B\rfloor\right\}
$$

where for a divisor $D=\sum d_{i} D_{i}$ we define $D^{\leq 1}=\sum d_{i}^{\prime} D_{i}$ with $d_{i}^{\prime}=\min \left\{d_{i}, 1\right\}$. In particular, $(B+\alpha M)^{\leq 1}=B+C$ for some $C \geq 0$ supported in Supp $M$, and $\alpha M=C+A$ where $A \geq 0$ is supported in $\lfloor B\rfloor$ and $C$ has no common components with $\lfloor B\rfloor$. Note that $\theta(X, B, M)$ is equal to the number of components of $C$. The pair $(X, B+C)$ is lc and the expression

$$
K_{X}+B+C \equiv P+M+C / Z
$$

is a weak Zariski decomposition $/ Z$. By construction

$$
\theta(X, B+C, M+C)<\theta(X, B, M)
$$

so $(X, B+C)$ has a log minimal model over $Z$ by minimality of $\theta(X, B, M)$ and the definition of $\mathfrak{W}$. Let $\left(Y,(B+C)_{Y}\right)$ be the minimal model.

Let $g: V \rightarrow X$ and $h: V \rightarrow Y$ be a common resolution. By definition, $K_{Y}+(B+C)_{Y}$ is nef $/ Z$. In particular, the expression

$$
g^{*}\left(K_{X}+B+C\right)=P^{\prime}+M^{\prime}
$$

is a weak Zariski decomposition $/ Z$ of $K_{X}+B+C$ where $P^{\prime}=h^{*}\left(K_{Y}+(B+C)_{Y}\right)$ and $M^{\prime} \geq 0$ is exceptional $/ Y$ (cf. [2, Remark $\left.2.4(2)\right]$ ). Moreover,

$$
g^{*}\left(K_{X}+B+C\right)=P^{\prime}+M^{\prime} \equiv g^{*} P+g^{*}(M+C) / Z
$$

Since $M^{\prime}$ is exceptional $/ Y$,

$$
h_{*}\left(g^{*}(M+C)-M^{\prime}\right) \geq 0
$$


On the other hand,

$$
g^{*}(M+C)-M^{\prime} \equiv P^{\prime}-g^{*} P / Z
$$

is anti-nef $/ Y$ hence by the negativity lemma, $g^{*}(M+C)-M^{\prime} \geq 0$. Therefore $\operatorname{Supp} M^{\prime} \subseteq \operatorname{Supp} g^{*}(M+C)=\operatorname{Supp} g^{*} M$.

Now,

$$
\begin{aligned}
(1+\alpha) g^{*}\left(K_{X}+B\right) & \equiv g^{*}\left(K_{X}+B\right)+\alpha g^{*} P+\alpha g^{*} M \\
& \equiv g^{*}\left(K_{X}+B\right)+\alpha g^{*} P+g^{*} C+g^{*} A \\
& \equiv P^{\prime}+\alpha g^{*} P+M^{\prime}+g^{*} A / Z
\end{aligned}
$$

hence we get a weak Zariski decomposition $/ Z$ as

$$
g^{*}\left(K_{X}+B\right) \equiv P^{\prime \prime}+M^{\prime \prime} / Z
$$

where

$$
P^{\prime \prime}=\frac{1}{1+\alpha}\left(P^{\prime}+\alpha g^{*} P\right) \quad \text { and } \quad M^{\prime \prime}=\frac{1}{1+\alpha}\left(M^{\prime}+g^{*} A\right)
$$

and $\operatorname{Supp} M^{\prime \prime} \subseteq \operatorname{Supp} g^{*} M$ hence $\operatorname{Supp} g_{*} M^{\prime \prime} \subseteq \operatorname{Supp} M$. Since $\theta(X, B, M)$ is minimal,

$$
\theta(X, B, M)=\theta\left(X, B, M^{\prime \prime}\right)
$$

So every component of $C$ is also a component of $g_{*} M^{\prime \prime}$ which in turn implies that every component of $C$ is also a component of $g_{*} M^{\prime}$. But $M^{\prime}$ is exceptional $/ Y$ hence so is $C$ which means that $(B+C)_{Y}=B^{\sim}+C^{\sim}+E=B^{\sim}+E=B_{Y}$ where $\sim$ stands for birational transform and $E$ is the reduced exceptional divisor of $Y \rightarrow X$. Thus we have $P^{\prime}=h^{*}\left(K_{Y}+B_{Y}\right)$. Although $K_{Y}+B_{Y}$ is nef $/ Z,\left(Y, B_{Y}\right)$ is not necessarily a $\log$ minimal model of $(X, B)$ over $Z$ because condition (4) of definition of log minimal models may not be satisfied (see 2.7).

Let $G$ be the largest $\mathbb{R}$-divisor such that $G \leq g^{*} C$ and $G \leq M^{\prime}$. By letting $\tilde{C}=g^{*} C-G$ and $\tilde{M}^{\prime}=M^{\prime}-G$ we get the expression

$$
g^{*}\left(K_{X}+B\right)+\tilde{C}=P^{\prime}+\tilde{M}^{\prime}
$$

where $\tilde{C}$ and $\tilde{M}^{\prime}$ are effective with no common components.

Assume that $\tilde{C}$ is exceptional $/ X$. Then $g^{*}\left(K_{X}+B\right)-P^{\prime}=\tilde{M}^{\prime}-\tilde{C}$ is antinef $/ X$ so by the negativity lemma $\tilde{M}^{\prime}-\tilde{C} \geq 0$ which implies that $\tilde{C}=0$ since $\tilde{C}$ and $\tilde{M}^{\prime}$ have no common components. Thus

$$
g^{*}\left(K_{X}+B\right)-h^{*}\left(K_{Y}+B_{Y}\right)=\sum_{D} a\left(D, Y, B_{Y}\right) D-a(D, X, B) D=\tilde{M}^{\prime}
$$

where $D$ runs over the prime divisors on $V$. If $\operatorname{Supp} g_{*} \tilde{M}^{\prime}=\operatorname{Supp} g_{*} M^{\prime}$, then Supp $\tilde{M}^{\prime}$ contains the birational transform of all the prime exceptional/ $Y$ divisors on $X$ hence $\left(Y, B_{Y}\right)$ is a log minimal model of $(X, B)$ over $Z$, a contradiction. Thus

$$
\operatorname{Supp}\left(g_{*} M^{\prime}-g_{*} G\right)=\operatorname{Supp} g_{*} \tilde{M}^{\prime} \subsetneq \operatorname{Supp} g_{*} M^{\prime} \subseteq \operatorname{Supp} M
$$


so some component of $C$ is not a component of $g_{*} \tilde{M}^{\prime}$ because $\operatorname{Supp} g_{*} G \subseteq$ Supp C. Therefore

$$
\theta(X, B, M)>\theta\left(X, B, \tilde{M}^{\prime}\right)
$$

which gives a contradiction again by minimality of $\theta(X, B, M)$ and the assumption that $(X, B)$ has no $\log$ minimal model over $Z$.

So we may assume that $\tilde{C}$ is not exceptional $/ X$. Let $\beta>0$ be the smallest number such that $\tilde{A}:=\beta g^{*} M-\tilde{C}$ satisfies $g_{*} \tilde{A} \geq 0$. Then there is a component of $g_{*} \tilde{C}$ which is not a component of $g_{*} \tilde{A}$. Now

$$
\begin{aligned}
(1+\beta) g^{*}\left(K_{X}+B\right) & \equiv g^{*}\left(K_{X}+B\right)+\beta g^{*} M+\beta g^{*} P \\
& \equiv g^{*}\left(K_{X}+B\right)+\tilde{C}+\tilde{A}+\beta g^{*} P \\
& \equiv P^{\prime}+\beta g^{*} P+\tilde{M}^{\prime}+\tilde{A} / Z
\end{aligned}
$$

where $\tilde{M}^{\prime}+\tilde{A} \geq 0$ by the negativity lemma. Thus we get a weak Zariski decomposition $/ Z$ as $g^{*}\left(K_{X}+B\right) \equiv P^{\prime \prime \prime}+M^{\prime \prime \prime} / Z$ where

$$
P^{\prime \prime \prime}=\frac{1}{1+\beta}\left(P^{\prime}+\beta g^{*} P\right) \quad \text { and } \quad M^{\prime \prime \prime}=\frac{1}{1+\beta}\left(\tilde{M}^{\prime}+\tilde{A}\right)
$$

and $\operatorname{Supp} g_{*} M^{\prime \prime \prime} \subseteq \operatorname{Supp} M$. Moreover, by construction, there is a component $D$ of $g_{*} \tilde{C}$ which is not a component of $g_{*} \tilde{A}$. Since $g_{*} \tilde{C} \leq C, D$ is a component of $C$ hence of $M$, and since $\tilde{C}$ and $\tilde{M}^{\prime}$ have no common components, $D$ is not a component of $g_{*} \tilde{M}^{\prime}$. Therefore $D$ is not a component of $g_{*} M^{\prime \prime \prime}=\frac{1}{1+\beta}\left(g_{*} \tilde{M}^{\prime}+\right.$ $\left.g_{*} \tilde{A}\right)$ which implies that

$$
\theta(X, B, M)>\theta\left(X, B, M^{\prime \prime \prime}\right)
$$

giving a contradiction again.

\subsection{Proofs of 1.2 and 1.1.}

Proof. (of Theorem 1.2) By applying Lemma 7.5, we can reduce the problem to the case when $X, Z$ are projective. We can find a $\log$ resolution $f: W \rightarrow X$ and a $\mathbb{Q}$-boundary $B_{W}$ such that

$$
K_{W}+B_{W}=f^{*}\left(K_{X}+B\right)+E
$$

where $E \geq 0$ and its support is equal to the union of the exceptional divisors of $f$, and $\left(W, B_{W}\right)$ has terminal singularities. It is enough to construct a log minimal model for $\left(W, B_{W}\right)$ over $Z$. So by replacing $(X, B)$ with $\left(W, B_{W}\right)$ we can assume $(X, B)$ has terminal singularities and that $X$ is $\mathbb{Q}$-factorial.

Let

$$
\mathcal{E}=\left\{B^{\prime} \mid K_{X}+B^{\prime} \text { is pseudo-effective } / Z \text { and } 0 \leq B^{\prime} \leq B\right\}
$$

which is a compact subset of the $\mathbb{R}$-vector space $V$ generated by the components of $B$. Let $B^{\prime}$ be an element in $\mathcal{E}$ which has minimal distance from 0 with respect to the standard metric on $V$. So either $B^{\prime}=0$, or $K_{X}+B^{\prime \prime}$ is not pseudo-effective $/ Z$ for any $0 \leq B^{\prime \prime} \lesseqgtr B^{\prime}$. 


\section{Existence of flips and minimal models for 3-folds in char $p$}

31

Run the generalized LMMP $/ Z$ on $K_{X}+B^{\prime}$ as follows [13, proof of Theorem 5.6]: let $R$ be a $K_{X}+B^{\prime}$-negative extremal ray/ $Z$. By 3.3, $R$ is either a divisorial extremal ray or a flipping extremal ray (see the beginning of Section 6 for definitions), and $R$ can be contracted to an algebraic space. If $R$ is a divisorial extremal ray, then it can actually be contracted by a projective morphism, by Lemma 7.2, and we continue the process. But if $R$ is a flipping extremal ray, then we use the generalized flip, which exists by Theorem 6.3, and then continue the process.

No component of $B^{\prime}$ is contracted by the LMMP: otherwise let $X_{i} \rightarrow X_{i+1}$ be the sequence of $\log$ flips and divisorial contractions of this LMMP where $X=X_{1}$. Pick $j$ so that $\phi_{j}: X_{j} \rightarrow X_{j+1}$ is a divisorial contraction which contracts a component $D_{j}$ of $B_{j}^{\prime}$, the birational transform of $B^{\prime}$. Now there is $a>0$ such that

$$
K_{X_{j}}+B_{j}^{\prime}=\phi_{j}^{*}\left(K_{X_{j+1}}+B_{j+1}^{\prime}\right)+a D_{j}
$$

Since $K_{X_{j+1}}+B_{j+1}^{\prime}$ is pseudo-effective $/ Z, K_{X_{j}}+B_{j}^{\prime}-a D_{j}$ is pseudo-effective $/ Z$ which implies that $K_{X}+B^{\prime}-b D$ is pseudo-effective/ $Z$ for some $b>0$ where $D$ is the birational transform of $D_{j}$, a contradiction. Therefore every $\left(X_{j}, B_{j}^{\prime}\right)$ has terminal singularities. The LMMP terminates for reasons similar to the characteristic 0 case [27, Corollary 2.17][21, Theorem 6.17] (see also [13, proof of Theorem 1.2]). So we get a $\log$ minimal model of $\left(X, B^{\prime}\right)$ over $Z$, say $\left(Y, B_{Y}^{\prime}\right)$.

Let $g: V \rightarrow X$ and $h: V \rightarrow Y$ be a common resolution. By letting $P=$ $h^{*}\left(K_{Y}+B_{Y}^{\prime}\right)$ and

$$
M=g^{*}\left(K_{X}+B\right)-h^{*}\left(K_{Y}+B_{Y}^{\prime}\right)
$$

we get a weak Zariski decomposition $/ Z$ as $g^{*}\left(K_{X}+B\right)=P+M / Z$. Note that $M \geq 0$ because $g^{*}\left(K_{X}+B^{\prime}\right)-h^{*}\left(K_{Y}+B_{Y}^{\prime}\right) \geq 0$. Therefore $(X, B)$ has a log minimal model over $Z$ by Proposition 8.3.

Proof. (of Theorem 1.1 in general case) Recall that we proved the theorem when $X$ is projective, in Section 6. By perturbing the coefficients, we can assume that $(X, B)$ is klt. By Theorem $1.2,(X, B)$ has a $\log$ minimal model over $Z$, say $\left(X^{+}, B^{+}\right)$. Since $(X, B)$ is klt, $X \rightarrow X^{+}$is an isomorphism in codimension one. Let $H^{+}$be an ample $/ Z$ divisor on $X^{+}$and let $H$ be its birational transform on $X$. Since $X \rightarrow Z$ is a $K_{X}+B$-negative extremal contraction, $K_{X}+B \equiv h H / Z$ for some $h>0$. Thus $K_{X^{+}}+B^{+} \equiv h H^{+} / Z$ which means that $K_{X^{+}}+B^{+}$is ample $/ Z$ so we are done.

\section{The CONnECtedness PRINCIPle With APPlications to SEMI-AMPLENESS}

9.1. Connectedness. In this subsection, we prove the connectedness principle in dimension $\leq 3$. The proof is based on LMMP rather than vanishing theorems.

The following lemma is essentially [30, Proposition 2.3]. We recall its proof for convenience. 
Lemma 9.2. Let $(X, B)$ be a projective pair of dimension $\leq 3$ over $k$. Assume that $(X, B)$ is klt (resp. dlt) and that $A$ is a nef and big (resp. ample) $\mathbb{R}$-divisor. Then there is $0 \leq A^{\prime} \sim_{\mathbb{R}} A$ such that $\left(X, B+A^{\prime}\right)$ is klt (resp. dlt).

Proof. First we deal with the dlt case. Let $f: W \rightarrow X$ be a log resolution of $(X, B)$ which extracts only prime divisors with positive log discrepancy with respect to $(X, B)$. This exists by the definition of dlt pairs. The resolution is obtained by a sequence of blow ups with smooth centers, hence there is an $\mathbb{R}$-divisor $E^{\prime}$ exceptional/ $X$ with sufficiently small coefficients such that $-E^{\prime}$ is ample/ $X$ and Supp $E^{\prime}$ is the union of all the prime exceptional/ $X$ divisors on $W$. Note that by the negativity lemma $(2.3), E^{\prime} \geq 0$. Moreover, $f^{*} A-E^{\prime}$ is ample/ $X$.

Let $B_{W}$ be given by

$$
K_{W}+B_{W}=f^{*}\left(K_{X}+B\right)
$$

By assumption, $B_{W}$ has coefficients at most 1 and the coefficient of any prime exceptional/ $X$ divisor is less than 1 . Let $A_{W}^{\prime} \sim_{\mathbb{R}} f^{*} A-E^{\prime}$ be general and let $A^{\prime}:=f_{*} A_{W}^{\prime}$. Then $A^{\prime} \sim_{\mathbb{R}} A$ and we can write

$$
K_{W}+B_{W}+A_{W}^{\prime}+E^{\prime}=f^{*}\left(K_{X}+B+A^{\prime}\right)
$$

where we can make sure that the coefficients of $B_{W}+A_{W}^{\prime}+E^{\prime}$ are at most 1 and that the coefficient of any prime exceptional/ $X$ divisor is less than 1 because the coefficients of $E^{\prime}$ are sufficiently small. This implies that $\left(X, B+A^{\prime}\right)$ is dlt.

Now we deal with the klt case. Since $A$ is nef and big, by definition, $A \sim_{\mathbb{R}}$ $G+D$ with $G \geq 0$ ample and $D \geq 0$. So by replacing $A$ with $(1-\epsilon) A+\epsilon G$ and replacing $B$ with $B+\epsilon D$ we can assume that $A$ is ample. Now apply the dlt case.

Proof. (of Theorem 1.8) Assume that the statement does not hold for some $z$. By Lemma 7.5, there is a $\mathbb{Q}$-factorial dlt pair $\left(Y, B_{Y}\right)$ and a birational morphism $g: Y \rightarrow X$ with $K_{Y}+B_{Y}$ nef $/ X$, every exceptional divisor of $g$ is a component of $\left\lfloor B_{Y}\right\rfloor$, and $g_{*} B_{Y}=B$. Moreover, $K_{Y}+B_{Y}+E_{Y}=f^{*}\left(K_{X}+B\right)$ for some $E_{Y} \geq 0$ with Supp $E_{Y} \subseteq\left\lfloor B_{Y}\right\rfloor$. Also the non-klt locus of $\left(Y, B_{Y}\right)$, that is $\left\lfloor B_{Y}\right\rfloor$, maps surjectively onto the non-klt locus of $(X, B)$ hence $\left\lfloor B_{Y}\right\rfloor$ is not connected in some neighborhood of $Y_{z}$.

Now by assumptions, $K_{Y}+B_{Y}+E_{Y}+L_{Y} \sim_{\mathbb{R}} 0 / Z$ for some globally nef and big $\mathbb{R}$-divisor $L_{Y}$. Since $X$ is $\mathbb{Q}$-factorial, we can write $L_{Y} \sim_{\mathbb{R}} A_{Y}+D_{Y}$ where $A_{Y}$ is ample and $D_{Y} \geq 0$ is exceptional $/ X$. In particular, Supp $D_{Y} \subset\left\lfloor B_{Y}\right\rfloor$. By picking a general

$$
G_{Y} \sim_{\mathbb{R}} \epsilon A_{Y}+(1-\epsilon) L_{Y}-\delta\left\lfloor B_{Y}\right\rfloor
$$

for some small $\delta>0$ and applying Lemma 9.2 we can assume that $\left(Y, B_{Y}+G_{Y}\right)$ is dlt. By construction,

$$
K_{Y}+B_{Y}+G_{Y} \sim_{\mathbb{R}} P_{Y}:=-\epsilon D_{Y}-E_{Y}-\delta\left\lfloor B_{Y}\right\rfloor / Z
$$

and Supp $P_{Y}=\left\lfloor B_{Y}\right\rfloor$. 
Run a generalized LMMP/Z on $K_{Y}+B_{Y}+G_{Y}$ as in the proof of Theorem 1.2. We show that this is actually a usual LMMP hence it terminates by special termination (5.5). Assume that we have arrived at a model $Y^{\prime}$ and let $R$ be a $K_{Y^{\prime}}+B_{Y^{\prime}}+G_{Y^{\prime}}$-negative extremal ray $/ Z$. Since $Y^{\prime} \rightarrow Z$ is birational, $R$ is either a divisorial extremal ray or a flipping extremal ray. In the former case $R$ can be contracted by a projective morphism by Lemma 7.2. So assume $R$ is a flipping extremal ray. Then the generalized flip $Y^{\prime} \rightarrow Y^{\prime \prime} / V$ exists by Theorem 6.3 where $Y^{\prime} \rightarrow V$ is the contraction of $R$ to the algebraic space $V$. Since $P_{Y^{\prime}} \cdot R<0$, some component $S_{Y^{\prime}}$ of $\left\lfloor B_{Y^{\prime}}\right\rfloor$ intersects $R$ positively. Now there is a boundary $\Delta_{Y^{\prime}}$ such that $\left(Y^{\prime}, \Delta_{Y^{\prime}}\right)$ is plt, $S_{Y^{\prime}}=\left\lfloor\Delta_{Y^{\prime}}\right\rfloor$, and $\left(K_{Y^{\prime}}+\Delta_{Y^{\prime}}\right) \cdot R=0$. But then we can find $N_{Y^{\prime \prime}} \geq 0$ such that $\left(Y^{\prime \prime}, \Delta_{Y^{\prime \prime}}+N_{Y^{\prime \prime}}\right)$ is plt and $\left(K_{Y^{\prime \prime}}+\Delta_{Y^{\prime \prime}}+N_{Y^{\prime \prime}}\right) \cdot R<0$. Therefore by 5.3 and $2.13, Y^{\prime \prime} \rightarrow V$ is a projective morphism which implies that $Y^{\prime} \rightarrow V$ is also a projective morphism and that the flip is a usual flip.

We claim that the connected components of $\left\lfloor B_{Y}\right\rfloor$ over $z$ remain disjoint over $z$ in the course of the LMMP: assume not and let $Y^{\prime}$ be the first model in the process such that there are irreducible components $S_{Y}, T_{Y}$ of $\left\lfloor B_{Y}\right\rfloor$ belonging to disjoint connected components over $z$ such that $S_{Y^{\prime}}, T_{Y^{\prime}}$ intersect over $z$. Let $\Delta_{Y}=B_{Y}-\tau\left(\left\lfloor B_{Y}\right\rfloor-S_{Y}-T_{Y}\right)$ for some small $\tau>0$. Then $\left(Y, \Delta_{Y}+G_{Y}\right)$ is plt in some neighborhood of $Y_{z}$ because $\left\lfloor\Delta_{Y}+G_{Y}\right\rfloor=S_{Y}+T_{Y}$ and $S_{Y}, T_{Y}$ are disjoint over $z$. Moreover, $Y \rightarrow Y^{\prime}$ is a partial LMMP on $K_{Y}+\Delta_{Y}+G_{Y}$ hence $\left(Y^{\prime}, \Delta_{Y^{\prime}}+G_{Y^{\prime}}\right)$ is also plt over $z$. But since $S_{Y^{\prime}}, T_{Y^{\prime}}$ intersect over $z$, $\left(Y^{\prime}, \Delta_{Y^{\prime}}+G_{Y^{\prime}}\right)$ cannot be plt over $z$, a contradiction.

Next we claim that no connected component of $\left\lfloor B_{Y}\right\rfloor$ over $z$ can be contracted by the LMMP (although some of their irreducible components might be contracted). By construction $-P_{Y} \geq 0$ and Supp $-P_{Y}=\left\lfloor B_{Y}\right\rfloor$, and $-P_{Y}$ is positive on each extremal ray in the LMMP. Write $-P_{Y}=\sum-P_{Y}^{i}$ where $-P_{Y}^{i}$ are the connected components of $-P_{Y}$ over $z$. By the previous paragraph, $-P_{Y}^{i}$ and $-P_{Y}^{j}$ remain disjoint during the LMMP if $i \neq j$. Moreover, if we arrive a model $Y^{\prime}$ in the LMMP on which we contract an extremal ray $R$, then $-P_{Y^{\prime}}^{j} \cdot R>0$ for some $j$ and $-P_{Y^{\prime}}^{i} \cdot R=0$ for $i \neq j$. Therefore the contraction of $R$ cannot contract any of the $-P_{Y^{\prime}}^{i}$.

The LMMP ends up with a $\log$ minimal model $\left(Y^{\prime}, B_{Y^{\prime}}+G_{Y^{\prime}}\right)$ over $Z$. Then $P_{Y^{\prime}}$ is nef $/ Z$. Assume that $Y_{z}^{\prime} \nsubseteq \operatorname{Supp} P_{Y^{\prime}}$ set-theoretically. Since $Y_{z}^{\prime}$ intersects Supp $P_{Y^{\prime}}$, there is some curve $C \subset Y_{z}^{\prime}$ not contained in Supp $P_{Y^{\prime}}$ but intersects it. Then as $-P_{Y^{\prime}} \geq 0$ we have $-P_{Y^{\prime}} \cdot C>0$ hence $P_{Y^{\prime}} \cdot C<0$, a contradiction. Now since $Y_{z}^{\prime}$ is connected, it is contained in exactly one connected component of $\left\lfloor B_{Y^{\prime}}\right\rfloor$ over $z$. This is a contradiction because by assumptions at least two connected components of $\left\lfloor B_{Y^{\prime}}\right\rfloor$ over $z$ intersect the fibre $Y_{z}^{\prime}$.

We now show that a strong form of the connectedness principle holds on surfaces.

Theorem 9.3. Let $(X, B)$ be a $\mathbb{Q}$-factorial projective pair of dimension 2 over $k$. Let $f: X \rightarrow Z$ be a projective contraction (not necessarily birational) such 
that $-\left(K_{X}+B\right)$ is ample $/ Z$. Then for any closed point $z \in Z$, the non-klt locus $N$ of $(X, B)$ is connected in any neighborhood of the fibre $X_{z}$ over $z$. More strongly, $N \cap X_{z}$ is connected.

Proof. It is enough to prove the last claim. Assume that $N \cap X_{z}$ is not connected for some $z$. We use the notation and the arguments of the proof of Theorem 1.8. Let $\left(Y, B_{Y}\right)$ be the pair constructed over $X$ and $Y \rightarrow Y^{\prime}$ the LMMP $/ Z$ on $K_{Y}+B_{Y}+G_{Y} \sim_{\mathbb{R}} P_{Y}$ and $h: Y^{\prime} \rightarrow Z$ the corresponding map. The same arguments of the proof of Theorem 1.8 show that the connected components of $P_{Y}$ over $z$ remain disjoint in the course of the LMMP and none of them will be contracted.

By assumptions, $\left\lfloor B_{Y}\right\rfloor \cap Y_{z}$ is not connected. We claim that the same holds in the course of the LMMP. If not, then at some step of the LMMP we arrive at a model $W$ with a $K_{W}+B_{W}+G_{W}$-negative extremal birational contraction $\phi: W \rightarrow V$ such that $\left\lfloor B_{W}\right\rfloor \cap W_{z}$ is not connected but $\left\lfloor B_{V}\right\rfloor \cap V_{z}$ is connected. Let $C$ be the exceptional curve of $W \rightarrow V$. Now $\phi\left(\left\lfloor B_{W}\right\rfloor\right)=\left\lfloor B_{V}\right\rfloor$ : the inclusion $\supseteq$ is clear; the inclusion $\subseteq$ follows from the fact that if $C$ is a component of $\left\lfloor B_{W}\right\rfloor$, then at least one other irreducible component of $\left\lfloor B_{W}\right\rfloor$ intersects $C$ because $P_{W} \cdot C<0$. Therefore $\phi\left(\left\lfloor B_{W}\right\rfloor \cap W_{z}\right)=\left\lfloor B_{V}\right\rfloor \cap V_{z}$. Since $\left\lfloor B_{V}\right\rfloor \cap V_{z}$ is connected but $\left\lfloor B_{W}\right\rfloor \cap W_{z}$ is not connected, there exist two connected components of $\left\lfloor B_{W}\right\rfloor \cap W_{z}$ whose images under $\phi$ intersect. So there are closed points $w, w^{\prime}$ belonging to different connected components of $\left\lfloor B_{W}\right\rfloor \cap W_{z}$ such that $\phi(w)=\phi\left(w^{\prime}\right)$. In particular, $w, w^{\prime} \in C$. Note that $C$ is not a component of $\left\lfloor B_{W}\right\rfloor$ otherwise $C \subset\left\lfloor B_{W}\right\rfloor \cap W_{z}$ connects $w, w^{\prime}$ which contradicts the assumptions. Therefore $\left\lfloor B_{W}\right\rfloor \cap C$ is a finite set of closed points with more than one element. Now perturbing the coefficients of $B_{W}$ we can find a $\Gamma_{W} \leq\left\lfloor B_{W}\right\rfloor$ such that $\left(W, \Gamma_{W}\right)$ is plt in a neighborhood of $C,\left(K_{W}+\Gamma_{W}\right) \cdot C<0$ and such that $\left\lfloor\Gamma_{W}\right\rfloor \cap C$ is a finite set of closed points with more than one element. Then in a formal neighborhood of $\phi(w),\left\lfloor\Gamma_{V}\right\rfloor$ has at least two branches which implies that $\left\lfloor\Gamma_{V}\right\rfloor$ is not normal which in turn contradicts the plt property of $\left(V, \Gamma_{V}\right)$.

Since $\left\lfloor B_{Y^{\prime}}\right\rfloor \cap Y_{z}^{\prime}$ is not connected, there is a component $D$ of $Y_{z}^{\prime}$ not contained in Supp $P_{Y^{\prime}}=\left\lfloor B_{Y^{\prime}}\right\rfloor$ but intersects it. Thus $P_{Y^{\prime}}$ cannot be nef $/ Z$ as $-P_{Y^{\prime}} \geq 0$. Therefore the LMMP terminates with a Mori fibre space $Y^{\prime} \rightarrow Z^{\prime} / Z$. If $Z^{\prime}$ is a point, then $\left\lfloor B_{Y^{\prime}}\right\rfloor$ has at least two disjoint irreducible components which contradicts the fact that the Picard number $\rho\left(Y^{\prime}\right)=1$ in this case. So we can assume that $Z^{\prime}$ is a curve.

Assume that $Z$ is also a curve in which case $Z^{\prime}=Z$. Let $F$ be the reduced variety associated to a general fibre of $Y^{\prime} \rightarrow Z^{\prime}$. Then by the adjunction formula we get $F \simeq \mathbb{P}^{1}, K_{Y^{\prime}} \cdot F=-2$, and $\left(B_{Y^{\prime}}+G_{Y^{\prime}}\right) \cdot F<2$. On the other hand, since $\left\lfloor B_{Y^{\prime}}\right\rfloor \cap Y_{z}^{\prime}$ has at least two points, $\left\lfloor B_{Y^{\prime}}\right\rfloor \cap F$ also has at least two points hence

$$
\left(B_{Y^{\prime}}+G_{Y^{\prime}}\right) \cdot F \geq\left(\left\lfloor B_{Y^{\prime}}\right\rfloor+G_{Y^{\prime}}\right) \cdot F>2
$$

which is a contradiction. Now assume that $Z$ is a point. Since $\left\lfloor B_{Y^{\prime}}\right\rfloor \cap Y_{z}^{\prime}$ is not connected, $\left\lfloor B_{Y^{\prime}}\right\rfloor$ has at least two disjoint connected components, say $M_{Y^{\prime}}, N_{Y^{\prime}}$. On the other hand, since $P_{Y^{\prime}} \cdot F<0$, we may assume that $M_{Y^{\prime}}$ intersects $F$ (hence $M_{Y^{\prime}}$ intersects every fibre of $Y^{\prime} \rightarrow Z^{\prime}$ ). If some component of $N_{Y^{\prime}}$ is 


\section{Existence of flips and minimal models for 3-folds in char $p$}

35

vertical $/ Z^{\prime}$, then $M_{Y^{\prime}}, N_{Y^{\prime}}$ intersect a contradiction. Thus each component of $N_{Y^{\prime}}$ is horizontal $/ Z^{\prime}$ hence they intersect each fibre of $Y^{\prime} \rightarrow Z^{\prime}$. But then we can get a contradiction as in the $Z^{\prime}=Z$ case.

9.4. Semi-ampleness. We use the connectedness principle on surfaces to prove some semi-ampleness results in dimension 2 and 3. These are not only interesting on their own but also useful for the proof of the finite generation (1.3).

Proof. (of Theorem 1.9) Let $S \leq\lfloor B\rfloor$ be a reduced divisor. Assume that $\left.\left(K_{X}+B+A\right)\right|_{S}$ is not semi-ample. We will derive a contradiction. We can assume that if $S^{\prime} \lesseqgtr S$ is any other reduced divisor, then $\left.\left(K_{X}+B+A\right)\right|_{S^{\prime}}$ is semi-ample. Note that $S$ cannot be irreducible by abundance for surfaces (cf. [28]). Using the ample divisor $A$ and applying Lemma 9.2, we can perturb the coefficients of $B$ so that we can assume $S=\lfloor B\rfloor$.

Let $T$ be an irreducible component of $S$ and let $S^{\prime}=S-T$. By assumptions, $\left.\left(K_{X}+B+A\right)\right|_{T}$ and $\left.\left(K_{X}+B+A\right)\right|_{S^{\prime}}$ are both semi-ample. Let $g: T \rightarrow Z$ be the projective contraction associated to $\left.\left(K_{X}+B+A\right)\right|_{T}$. By adjunction define $K_{T}+B_{T}:=\left.\left(K_{X}+B\right)\right|_{T}$ and $A_{T}=\left.A\right|_{T}$. Since $K_{T}+B_{T}+A_{T} \sim_{\mathbb{Q}} 0 / Z$ and since $A_{T}$ is ample, $-\left(K_{T}+B_{T}\right)$ is ample/Z. Moreover, $S^{\prime} \cap T=\left\lfloor B_{T}\right\rfloor$ as topological spaces. By the connectedness principle for surfaces (9.3), $\left\lfloor B_{T}\right\rfloor \rightarrow Z$ has connected fibres hence $S^{\prime} \cap T \rightarrow Z$ also has connected fibres. Now apply Keel [16, Corollary 2.9].

Theorem 9.5. Let $(X, B+A)$ be a projective $\mathbb{Q}$-factorial dlt pair of dimension 3 over $k$ of char $p>5$. Assume that

- $A, B \geq 0$ are $\mathbb{Q}$-divisors with $A$ ample,

- $\left(Y, B_{Y}+A_{Y}\right)$ is a $\mathbb{Q}$-factorial weak lc model of $(X, B+A)$,

- $Y \rightarrow X$ does not contract any divisor,

- Supp $A_{Y}$ does not contain any lc centre of $\left(Y, B_{Y}+A_{Y}\right)$,

- if $\Sigma$ is a connected component of $\mathbb{E}\left(K_{Y}+B_{Y}+A_{Y}\right)$ and $\Sigma \nsubseteq\left\lfloor B_{Y}\right\rfloor$, then $\left.\left(K_{Y}+B_{Y}+A_{Y}\right)\right|_{\Sigma}$ is semi-ample.

Then $K_{Y}+B_{Y}+A_{Y}$ is semi-ample.

Proof. Note that if $K_{X}+B+A$ is not big, then $\mathbb{E}\left(K_{Y}+B_{Y}+A_{Y}\right)=Y$ hence the statement is trivial. So we can assume that $K_{X}+B+A$ is big. Let $\phi$ denote the map $X \rightarrow Y$ and let $U$ be the largest open set over which $\phi$ is an isomorphism. Then since $A$ is ample and $X$ is $\mathbb{Q}$-factorial, Supp $A_{Y}$ contains $Y \backslash \phi(U)$ : indeed let $y \in Y \backslash \phi(U)$ be a closed point and let $W$ be the normalization of the graph of $\phi$, and $\alpha: W \rightarrow X$ and $\beta: W \rightarrow Y$ be the corresponding morphisms; first assume that $\operatorname{dim} \beta^{-1}\{y\}>0$; then $\alpha^{*} A$ intersects $\beta^{-1}\{y\}$ because $A$ is ample hence $\operatorname{Supp} A_{Y}$ contains $y$; now assume that $\operatorname{dim} \beta^{-1}\{y\}=0$; then $\beta$ is an isomorphism over $y$; on the other hand, $\alpha$ cannot be an isomorphism near $\beta^{-1}\{y\}$ otherwise $\phi$ would be an isomorphism near $\alpha\left(\beta^{-1}\{y\}\right)$ hence $y \in \phi(U)$, a contradiction; thus as $X$ is $\mathbb{Q}$-factorial, $\alpha$ contracts some prime divisor $E$ containing $\beta^{-1}\{y\}$; but then $Y \rightarrow X$ contracts a divisor, a contradiction. 
Let $C \geq 0$ be any $\mathbb{Q}$-divisor such that $(X, B+A+C)$ is dlt. Then $\left(Y, B_{Y}+\right.$ $\left.A_{Y}+\epsilon C_{Y}\right)$ is dlt for any sufficiently small $\epsilon>0$ because $\left(Y, B_{Y}+A_{Y}\right)$ has no lc centre inside $Y \backslash \phi(U) \subset \operatorname{Supp} A_{Y}$. Now let $G_{Y} \geq 0$ be a general small ample $\mathbb{Q}$-divisor on $Y$ and $G$ its birational transform on $X$. Since $G$ is small, $A-G$ is ample. Let $C \sim_{\mathbb{Q}} A-G$ be a general $\mathbb{Q}$-divisor. Let

$$
\Gamma_{Y}:=B_{Y}+(1-\epsilon) A_{Y}+\epsilon C_{Y}+\epsilon G_{Y}
$$

Then

$$
K_{Y}+\Gamma_{Y} \sim_{\mathbb{Q}} K_{Y}+B_{Y}+A_{Y}
$$

and $\left\lfloor B_{Y}\right\rfloor=\left\lfloor\Gamma_{Y}\right\rfloor$. Moreover, by the above remarks and by Lemma 9.2 we can assume that $\left(Y, \Gamma_{Y}\right)$ is dlt.

Now by Theorem 1.9, $\left.\left(K_{Y}+\Gamma_{Y}\right)\right|_{\left\lfloor\Gamma_{Y}\right\rfloor}$ is semi-ample hence $\left.\left(K_{Y}+B_{Y}+A_{Y}\right)\right|_{\left\lfloor B_{Y}\right\rfloor}$ is semi-ample. Therefore $\left.\left(K_{Y}+B_{Y}+A_{Y}\right)\right|_{\Sigma}$ is semi-ample for any connected component of $\mathbb{E}\left(K_{Y}+B_{Y}+A_{Y}\right)$ hence we can apply Theorem 2.9.

\section{Finite Generation AND BASE POINT FREENESS}

10.1. Finite generation. In this subsection we prove Theorem 1.3.

Lemma 10.2. Let $(X, B)$ be a pair and $M$ a $\mathbb{Q}$-divisor satisfying the following properties:

(1) $(X, \operatorname{Supp}(B+M))$ is projective log smooth of dimension 3 over $k$ of char $p>5$,

(2) $K_{X}+B$ is a big $\mathbb{Q}$-divisor,

(3) $K_{X}+B \sim_{\mathbb{Q}} M \geq 0$ and $\lfloor B\rfloor \subset \operatorname{Supp} M \subseteq \operatorname{Supp} B$,

(4) $M=A+D$ where $A$ is an ample $\mathbb{Q}$-divisor and $D \geq 0$,

(5) $\alpha M=N+C$ for some rational number $\alpha>0$ such that $N, C \geq 0$ are $\mathbb{Q}$-divisors, $\operatorname{Supp} N=\lfloor B\rfloor$, and $(X, B+C)$ is dlt,

(6) there is an ample $\mathbb{Q}$-divisor $A^{\prime} \geq 0$ such that $A^{\prime} \leq A$ and $A^{\prime} \leq C$.

If $(X, B+t C)$ has an lc model for some real number $t \in(0,1]$, then $(X, B+$ $(t-\epsilon) C)$ also has an lc model for any sufficiently small $\epsilon>0$.

Proof. We can assume that $C \neq 0$. If we let $\Delta=B-\delta(N+C)$ for some small rational number $\delta>0$, then $(X, \Delta)$ is klt and $K_{X}+B$ is a positive multiple of $K_{X}+\Delta$ up to $\mathbb{Q}$-linear equivalence. Similarly, for any $s \in(0,1]$, there is $s^{\prime} \in(0, s)$ such that $\left(X, \Delta+s^{\prime} C\right)$ is klt and $K_{X}+B+s C$ is a positive multiple of $K_{X}+\Delta+s^{\prime} C$ up to $\mathbb{Q}$-linear equivalence. So if $\left(Y, \Delta_{Y}+s^{\prime} C_{Y}\right)$ is a log minimal model of $\left(X, \Delta+s^{\prime} C\right)$, which exists by Theorem 1.2 , then $\left(Y, B_{Y}+s C_{Y}\right)$ is a $\mathbb{Q}$-factorial weak lc model of $(X, B+s C)$ such that $Y \rightarrow X$ does not contract divisors and $X \rightarrow Y$ is $K_{X}+B+s C$-negative (see 2.2 for this notion). We will make use of this observation below.

Let $T$ be the lc model of $(X, B+t C)$ and let $\left(Y, B_{Y}+t C_{Y}\right)$ be a $\mathbb{Q}$-factorial weak lc model of $(X, B+t C)$ such that $X \rightarrow Y$ is $K_{X}+B+t C$-negative and its inverse does not contract divisors. Then the induced map $Y \rightarrow T$ is a morphism and $K_{T}+B_{T}+t C_{T}$ pulls back to $K_{Y}+B_{Y}+t C_{Y}$. 


\section{Existence of flips and minimal models for 3-folds in char $p \quad 37$}

First assume that $t$ is irrational. Then $C_{Y} \equiv 0 / T$. Moreover, $C_{T}$ is $\mathbb{Q}$-Cartier because the set of those $s \in \mathbb{R}$ such that $K_{T}+B_{T}+s C_{T}$ is $\mathbb{R}$-Cartier forms a rational affine subspace of $\mathbb{R}$ (this can be proved using simple linear algebra similar to 3.4). Since $t$ belongs to this affine subspace and $t$ is not rational, the affine subspace is equal to $\mathbb{R}$ hence $K_{T}+B_{T}+s C_{T}$ is $\mathbb{R}$-Cartier for every $s$ which implies that $C_{T}$ is $\mathbb{Q}$-Cartier. Thus $C_{Y} \sim_{\mathbb{Q}} 0 / T$ hence $K_{T}+B_{T}+(t-\epsilon) C_{T}$ pulls back to $K_{Y}+B_{Y}+(t-\epsilon) C_{Y}$ and the former is ample for every sufficiently small $\epsilon>0$. This means that $T$ is also the lc model of $(X, B+(t-\epsilon) C)$.

From now on we assume that $t$ is rational. Replace $Y$ with a $\mathbb{Q}$-factorial weak lc model of $\left(Y, B_{Y}+(t-\epsilon) C_{Y}\right)$ over $T$ so that $X \rightarrow Y$ is still $K_{X}+B+$ $(t-\epsilon) C$-negative. Since $K_{T}+B_{T}+t C_{T}$ is ample, by choosing $\epsilon$ to be small enough, we can assume that $K_{Y}+B_{Y}+(t-\epsilon) C_{Y}$ is nef globally, by 3.3. Then $\left(Y, B_{Y}+(t-\epsilon) C_{Y}\right)$ is a weak lc model of $(X, B+(t-\epsilon) C)$ hence it is enough to show that $K_{Y}+B_{Y}+(t-\epsilon) C_{Y}$ is semi-ample. Perhaps after replacing $\epsilon$ with a smaller number we can assume that $K_{Y}+B_{Y}+\left(t-\epsilon^{\prime}\right) C_{Y}$ also nef globally for some $\epsilon^{\prime}>\epsilon$ and that $t-\epsilon$ is rational.

Let $Y \rightarrow V$ be the contraction to an algebraic space associated to $K_{Y}+B_{Y}+$ $(t-\epsilon) C_{Y}$. Any curve contracted by $Y \rightarrow V$ is also contracted by $Y \rightarrow T$ because $K_{Y}+B_{Y}+t C_{Y}$ and $K_{Y}+B_{Y}+\left(t-\epsilon^{\prime}\right) C_{Y}$ are both nef and $\epsilon^{\prime}>\epsilon$. Thus we get an induced map $V \rightarrow T$. Moreover, there is a small contraction $Y^{\prime} \rightarrow V$ from a $\mathbb{Q}$-factorial normal projective variety $Y^{\prime}$ : recall that $\left(Y, \Lambda_{Y}:=\Delta_{Y}+t^{\prime} C_{Y}\right)$ is klt where $\Delta$ and $t^{\prime}$ are as in the first paragraph; now $Y^{\prime}$ can be obtained by taking a log resolution $W \rightarrow Y$, defining $\Lambda_{W}$ to be the birational transform of $\Lambda_{V}$ plus the reduced exceptional divisor of $W \rightarrow V$, running an LMMP $/ V$ on $K_{W}+\Lambda_{W}$, using special termination and the fact that $K_{W}+\Lambda_{W} \equiv E / V$ for some $E \geq 0$ whose support is equal to the reduced exceptional divisor of $W \rightarrow V$, and applying the negativity lemma (2.3). Since $K_{Y}+B_{Y}+(t-\epsilon) C_{Y} \equiv 0 / V$, $\left.K_{Y^{\prime}}+B_{Y^{\prime}}+(t-\epsilon) C_{Y^{\prime}}\right)$ is also nef and the former is semi-ample if and only if the latter is. So by replacing $Y$ with $Y^{\prime}$, we can in addition assume that $Y \rightarrow V$ is a small contraction.

Let $\Sigma$ be a connected component of the exceptional set of $Y \rightarrow V$. Since $Y \rightarrow V$ is a small morphism, $\Sigma$ is one-dimensional. On the other hand, since

$$
K_{Y}+B_{Y}+(t-\epsilon) C_{Y} \equiv 0 / V
$$

and

$$
K_{Y}+B_{Y}+t C_{Y} \equiv 0 / V
$$

we get $C_{Y} \equiv 0 / V$ hence $N_{Y} \equiv 0 / V$. Therefore either $\Sigma \subset \operatorname{Supp} N_{Y}$ or $\Sigma \cap$ $\operatorname{Supp} N_{Y}=\emptyset$. Moreover, if $\Sigma \cap \operatorname{Supp} N_{Y}=\emptyset$, then $\left.\left(K_{Y}+B_{Y}+(t-\epsilon) C_{Y}\right)\right|_{\Sigma}$ is semi-ample because near $\Sigma$ the divisor $K_{Y}+B_{Y}+(t-\epsilon) C_{Y}$ is a multiple of $K_{Y}+B_{Y}+t C_{Y}$ and the latter is semi-ample.

We can assume that $A^{\prime}$ in (6) has small coefficients. Let $B^{\prime}=B+(t-\epsilon) C-A^{\prime}$. Since $\left(Y, B_{Y}^{\prime}+A_{Y}^{\prime}+\epsilon C_{Y}\right)$ is lc, Supp $C_{Y}$ (hence also Supp $A_{Y}^{\prime}$ ) does not contain any lc centre of $\left(Y, B_{Y}^{\prime}+A_{Y}^{\prime}\right)$. Now applying Theorem 9.5 to $\left(X, B^{\prime}+A^{\prime}\right)$ shows that $K_{Y}+B_{Y}+(t-\epsilon) C_{Y}$ is semi-ample (note that the exceptional locus of $Y \rightarrow V$ is equal to $\left.\mathbb{E}\left(K_{Y}+B_{Y}^{\prime}+A_{Y}^{\prime}\right)\right)$. Therefore, $K_{Y}+B_{Y}+s C_{Y}$ is semi-ample 
for every $s \in[t-\epsilon, t]$.

Proposition 10.3. Let $(X, B)$ be a pair and $M$ a $\mathbb{Q}$-divisor satisfying properties (1) to (4) of Lemma 10.2. Then the lc ring $R\left(K_{X}+B\right)$ is finitely generated.

Proof. Step 1. We follow the proof of [3, Proposition 3.4], which is similar to $[5, \S 5]$, but with some twists. Assume that $R\left(K_{X}+B\right)$ is not finitely generated. We will derive a contradiction. By replacing $A$ with $\frac{1}{m} S$ where $m$ is sufficiently divisible and $S$ is a general member of $|m A|$, and changing $M, B$ accordingly, we can assume that

(7) $S:=\operatorname{Supp} A$ is irreducible and $K_{X}+S+\Delta$ is ample for any boundary $\Delta$ supported on $\operatorname{Supp}(B)-S$.

Let $\theta(X, B, M)$ be the number of those components of $M$ which are not components of $\lfloor B\rfloor$ (such $\theta$ functions were defined in 8.1 in a more general setting). By (7), $S$ is not a component of $\lfloor B\rfloor$, hence $\theta(X, B, M)>0$ otherwise $K_{X}+B$ is ample and $R\left(K_{X}+B\right)$ is finitely generated, a contradiction. Define

$$
\alpha:=\min \left\{t>0 \mid\left\lfloor(B+t M)^{\leq 1}\right\rfloor \neq\lfloor B\rfloor\right\}
$$

where for a divisor $R=\sum r_{i} R_{i}$ we define $R^{\leq 1}=\sum r_{i}^{\prime} R_{i}$ with $r_{i}^{\prime}=\min \left\{r_{i}, 1\right\}$. In particular, $(B+\alpha M)^{\leq 1}=B+C$ for some $C \geq 0$ supported in $\operatorname{Supp} M$, and $\alpha M=C+N$ where $N \geq 0$ is supported in $\lfloor B\rfloor$ and $C$ has no common components with $\lfloor B\rfloor$.

Property (3) ensures that $\operatorname{Supp} N=\lfloor B\rfloor$, and by property (7) we have $\alpha A \leq$ $C$. So $(X, B)$ and $M$ also satisfy properties (5) and (6) of 10.2 with $A^{\prime}=\alpha^{\prime} A$ for some $\alpha^{\prime}>0$.

Step 2. Let $B^{\prime}:=B+C$ and let $M^{\prime}:=M+C$. Then the pair $\left(X, B^{\prime}\right)$ is $\log$ smooth dlt and

$$
\theta\left(X, B^{\prime}, M^{\prime}\right)<\theta(X, B, M)
$$

Assume that $R\left(K_{X}+B^{\prime}\right)$ is not finitely generated. By $(7), S$ is not a component of $\left\lfloor B^{\prime}\right\rfloor$ and $\theta\left(X, B^{\prime}, M^{\prime}\right)>0$. Now replace $(X, B)$ with $\left(X, B^{\prime}\right)$, replace $D$ with $D^{\prime}:=D+C$, and replace $M$ with $M^{\prime}$. By construction, all the properties (1) to (4) of 10.2 and property (7) above are still satisfied. Repeating the above process we get to the situation in which either $R\left(K_{X}+B^{\prime}\right)$ is finitely generated, or $\theta\left(X, B^{\prime}, M^{\prime}\right)=0$ and $K_{X}+B^{\prime}$ is ample. Thus in any case we can assume $R\left(K_{X}+B^{\prime}\right)$ is finitely generated.

Step 3. Let

$$
\mathcal{T}=\{t \in[0,1] \mid(X, B+t C) \text { has an lc model }\}
$$

Since $R\left(K_{X}+B^{\prime}=K_{X}+B+C\right)$ is finitely generated, $1 \in \mathcal{T}$ hence $\mathcal{T} \neq \emptyset$.

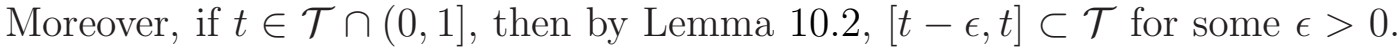
Now let $\tau=\inf \mathcal{T}$. If $\tau \in \mathcal{T}$, then $\tau=0$ which implies that $R\left(K_{X}+B\right)$ is finitely generated, a contradiction. So we may assume $\tau \notin \mathcal{T}$. There is a sequence $t_{1}>t_{2}>\cdots$ of rational numbers in $\mathcal{T}$ approaching $\tau$. For each $i$, there is a $\mathbb{Q}$-factorial weak lc model $\left(Y_{i}, B_{Y_{i}}+t_{i} C_{Y_{i}}\right)$ of $\left(X, B+t_{i} C\right)$ such that $Y_{i} \rightarrow X$ does not contract divisors (see the beginning of the proof of Lemma 


\section{Existence of flips and minimal models for 3-folds in char $p$}

10.2). By taking a subsequence, we can assume that all the $Y_{i}$ are isomorphic in codimension one. In particular, $N_{\sigma}\left(K_{Y_{1}}+B_{Y_{1}}+\tau C_{Y_{1}}\right)=0$.

Arguing as in the proof of Theorem 9.5, we can show that $\left(Y_{1}, B_{Y_{1}}+\tau C_{Y_{1}}\right)$ is dlt because $\alpha A \leq C$ is ample and Supp $A_{Y_{1}}$ does not contain any lc centre of $\left(Y_{1}, B_{Y_{1}}+\tau C_{Y_{1}}\right)$. Run the LMMP on $K_{Y_{1}}+B_{Y_{1}}+\tau C_{Y_{1}}$ with scaling of $\left(t_{1}-\tau\right) C_{Y_{1}}$ as in 3.5. Since $\alpha M_{Y_{1}}=N_{Y_{1}}+C_{Y_{1}}$, the LMMP is also an LMMP on $N_{Y_{1}}$. Thus each extremal ray in the process is a pl-extremal ray hence they can be contracted by projective morphisms (5.3). Moreover, the required flips exist by Theorem 1.1, and the LMMP terminates with a model $Y$ on which $K_{Y}+B_{Y}+\tau C_{Y}$ is nef, by special termination (5.5). Note that the LMMP does not contract any divisor by the $N_{\sigma}=0$ property. Moreover, $K_{Y}+B_{Y}+(\tau+\delta) C_{Y}$ is nef for some $\delta>0$. Now, by replacing the sequence we can assume that $K_{Y}+B_{Y}+t_{i} C_{Y}$ is nef for every $i$ and by replacing each $Y_{i}$ with $Y$ we can assume that $Y_{i}=Y$ for every $i$. A simple comparison of discrepancies (cf. $\left[3\right.$, Claim 3.5]) shows that $\left(Y, B_{Y}+\tau C_{Y}\right)$ is a $\mathbb{Q}$-factorial weak lc model of $(X, B+\tau C)$.

Step 4. Let $T_{i}$ be the lc model of $\left(X, B+t_{i} C\right)$. Then the map $Y \rightarrow \rightarrow T_{i}$ is a morphism and $K_{Y}+B_{Y}+t_{i} C_{Y}$ is the pullback of an ample divisor on $T_{i}$. Moreover, for each $i$, the map $T_{i+1} \rightarrow T_{i}$ is a morphism because any curve contracted by $Y \rightarrow T_{i+1}$ is also contracted by $Y \rightarrow T_{i}$. So perhaps after replacing the sequence, we can assume that $T_{i}$ is independent of $i$ so we can drop the subscript and simply use $T$. Since $C \sim_{\mathbb{Q}} 0 / T$, we can replace $Y$ with a $\mathbb{Q}$-factorialization of $T$ so that we can assume that $Y \rightarrow T$ is a small morphism (such a $\mathbb{Q}$-factorialization exists by the observations in the first paragraph of the proof of Lemma 10.2).

Assume that $\tau$ is irrational. If $K_{Y}+B_{Y}+(\tau-\epsilon) C_{Y}$ is nef for some $\epsilon>0$, then $K_{Y}+B_{Y}+\tau C_{Y}$ is semi-ample because in this case $K_{T}+B_{T}+(\tau-\epsilon) C_{T}$ is nef and $K_{T}+B_{T}+t_{i} C_{T}$ is ample hence $K_{T}+B_{T}+\tau C_{T}$ is ample. If there is no $\epsilon$ as above, then by 3.4 and 3.3 , there is a curve $\Gamma$ generating some extremal ray such that $\left(K_{Y}+B_{Y}+\tau C_{Y}\right) \cdot \Gamma=0$ and $C_{Y} \cdot \Gamma>0$. This is not possible since $\tau$ is assumed to be irrarional. So from now on we assume that $\tau$ is rational.

Step 5. Let $Y \rightarrow V$ be the contraction to an algebraic space associated to $K_{Y}+B_{Y}+\tau C_{Y}$. This map factors through $Y \rightarrow T$ so we get an induced map $T \rightarrow V$. We can write

$$
K_{T}+B_{T}+\tau C_{T}=a\left(K_{T}+B_{T}+t_{i} C_{T}\right)+b N_{T}
$$

for some $i$ and some rational numbers $a, b>0$. Since $K_{T}+B_{T}+t_{i} C_{T}$ is ample, we get

$$
\mathbb{E}\left(K_{T}+B_{T}+\tau C_{T}\right) \subset \operatorname{Supp} N_{T}=\left\lfloor B_{T}\right\rfloor
$$

Thus since $N_{Y} \sim_{\mathbb{Q}} 0 \sim_{\mathbb{Q}} C_{Y} / T$, the locus $\mathbb{E}\left(K_{Y}+B_{Y}+\tau C_{Y}\right)$ is a subset of the union of $\operatorname{Supp} N_{Y}=\left\lfloor B_{Y}\right\rfloor$ and the exceptional set of $Y \rightarrow T$. Let $\Lambda$ be a connected component of the exceptional set of $Y \rightarrow T$. Then, since $N_{Y} \sim_{\mathbb{Q}} 0 / T$ and since $\Lambda$ is one-dimensional, either $\Lambda \subset \operatorname{Supp} N_{Y}$ or $\Lambda \cap \operatorname{Supp} N_{Y}=\emptyset$. Therefore if $\Sigma$ is a connected component of $\mathbb{E}\left(K_{Y}+B_{Y}+\tau C_{Y}\right)$, then either $\Sigma \subset \operatorname{Supp} N_{Y}$ or $\Sigma \cap \operatorname{Supp} N_{Y}=\emptyset$. In the latter case, $\left.\left(K_{Y}+B_{Y}+\tau C_{Y}\right)\right|_{\Sigma}$ 
is semi-ample because near $\Sigma$ the divisor $K_{Y}+B_{Y}+\tau C_{Y}$ is a multiple of $K_{Y}+B_{Y}+t_{i} C_{Y}$ and the latter is semi-ample. Finally as in the end of the proof of Lemma 10.2 we can apply Theorem 9.5 to show that $K_{Y}+B_{Y}+\tau C_{Y}$ is semi-ample. This is a contradiction because we assumed $\tau \notin \mathcal{T}$.

Proof. (of Theorem 1.3) First assume that $Z$ is a point. Pick $M \geq 0$ such that $K_{X}+B \sim_{\mathbb{Q}} M$. We can choose $M$ so that $M=A+D$ where $A \geq 0$ is ample and $D \geq 0$. Let $f: W \rightarrow X$ be a $\log$ resolution of $(X, \operatorname{Supp}(B+M))$. Since $(X, B)$ is klt, we can write

$$
K_{W}+B_{W}=f^{*}\left(K_{X}+B\right)+E
$$

where $\left(W, B_{W}\right)$ is klt, $K_{W}+B_{W}$ is a $\mathbb{Q}$-divisor, and $E \geq 0$ is exceptional $/ X$. Moreover, there is $E^{\prime} \geq 0$ exceptional/ $X$ such that $-E^{\prime}$ is ample/ $X$ (cf. proof of Lemma 9.2). Let $A_{W} \sim_{\mathbb{Q}} f^{*} A-E^{\prime}$ be general and let $D_{W}=f^{*} D+E+E^{\prime}$. Then

$$
K_{W}+B_{W} \sim_{\mathbb{Q}} M_{W}:=A_{W}+D_{W}
$$

Now replace $(X, B)$ with $\left(W, B_{W}\right)$, replace $M$ with $M_{W}$, and replace $A$ and $D$ with $A_{W}$ and $D_{W}$. Moreover, by adding a small multiple of $M$ to $B$ we can also assume that $\operatorname{Supp} M \subseteq \operatorname{Supp} B$. Then $(X, B)$ and $M$ satisfy the properties (1) to (4) of Lemma 10.2. Therefore, by Proposition 10.3, $R\left(K_{X}+B\right)$ is finitely generated.

Now we treat the general case, that is, when $Z$ is not necessarily a point. By taking projectivizations of $X, Z$ and taking a log resolution, we may assume that $X, Z$ are projective and that $(X, B)$ is log smooth. We can also assume that $K_{X}+B \sim_{\mathbb{Q}} M=A+D / Z$ where $A$ is an ample $\mathbb{Q}$-divisor and $D \geq 0$. By adding some multiple of $M$ to $B$ we may assume Supp $M \subseteq \operatorname{Supp} B$. Let $\left(Y, B_{Y}\right)$ be a $\log$ minimal model of $(X, B)$ over $Z$. Let $H$ be the pullback of an ample divisor on $Z$. Since $A \leq B$, for each integer $m \geq 0$, there is $\Delta$ such that $K_{X}+B+m H \sim_{\mathbb{Q}} K_{X}+\Delta$ is big globally and that $(X, \Delta)$ is klt. Moreover, $\left(Y, \Delta_{Y}\right)$ is a $\log$ minimal model of $(X, \Delta)$ over $Z$. Now by 3.3 , if $m \gg 0$, then $K_{Y}+\Delta_{Y}$ is big and globally nef. On the other hand, $R\left(K_{Y}+\Delta_{Y}\right)$ is finitely generated over $k$ which means that $K_{Y}+\Delta_{Y}$ is semi-ample. Therefore $K_{Y}+B_{Y}$ is semi-ample $/ Z$ hence $\mathcal{R}\left(K_{X}+B / Z\right)$ is a finitely generated $\mathcal{O}_{Z}$-algebra.

\subsection{Base point freeness.}

Proof. (of Theorem 1.4) It is enough to show that $\mathcal{R}(D / Z)$ is a finitely generated $\mathcal{O}_{Z}$-algebra. By taking a $\mathbb{Q}$-factorialization using Theorem 1.6, we may assume that $X$ is $\mathbb{Q}$-factorial. Let $A=D-\left(K_{X}+B\right)$ which is nef and big $/ Z$ by assumptions. By replacing $A$, and replacing $B$ accordingly, we may assume that $A$ is ample globally. By Lemma 9.2, we can change $A$ up to $\mathbb{Q}$-linear equivalence so that $(X, B+A)$ is klt. But then $\mathcal{R}\left(K_{X}+B+A / Z\right)$ is finitely generated by Theorem 1.3 hence $\mathcal{R}(D / Z)$ is also finitely generated. 


\section{Existence of flips and minimal models for 3-folds in char $p \quad 41$}

\subsection{Contractions.}

Proof. (of Theorem 1.5) We may assume that $B$ is a $\mathbb{Q}$-divisor and that $(X, B)$ is klt. We can assume $N=H+D$ where $H$ is ample/ $Z$ and $D \geq 0$. Let $G$ be the pullback of an ample divior on $Z$, and let $N^{\prime}=m G+n N+\epsilon H+\epsilon D$ where $\epsilon>0$ is sufficiently small and $m \gg n \gg 0$. Then we can find $A \sim_{\mathbb{Q}} N^{\prime}$ such that $(X, B+A)$ is klt, $K_{X}+B+A$ is globally big, and $\left(K_{X}+B+A\right) \cdot R<0$. By 3.3, we can find an ample divisor $E$ such that $L:=\left(K_{X}+B+A+E\right)$ is nef and big globally and $L^{\perp}=R$. We can also assume that $(X, B+A+E)$ is klt hence by Theorem 1.4, $L$ is semi-ample which implies that $R$ can be contracted by a projective morphism.

\section{ACC FOR LC THRESHOLDS}

In this section, we prove Theorem 1.10 by a method similar to the characteristic 0 case (see [20, Chapter 18] and [22]). Let us recall the definition of $l c$ threshold. Let $(X, B)$ be an lc pair over $k$ and $M \geq 0$ an $\mathbb{R}$-Cartier divisor. The lc threshold of $M$ with respect to $(X, B)$ is defined as

$$
\operatorname{lct}(M, X, B)=\sup \{t \mid(X, B+t M) \text { is lc }\}
$$

We first prove some results, including ACC for lc thresholds, for surfaces before we move on to 3 -folds.

\subsection{ACC for lc thresholds on surfaces.}

Proposition 11.2. ACC for lc thresholds holds in dimension 2 (formulated similar to 1.10).

Proof. If this is not the case, then there is a sequence $\left(X_{i}, B_{i}\right)$ of lc pairs of dimension 2 over $k$ and $\mathbb{R}$-Cartier divisors $M_{i} \geq 0$ such that the coefficients of $B_{i}$ are in $\Lambda$, the coefficients of $M_{i}$ are in $\Gamma$ but such that the $t_{i}:=\operatorname{lct}\left(M_{i}, X_{i}, B_{i}\right)$ form a strictly increasing sequence of numbers. If for infinitely many $i,\left(X_{i}, \Delta_{i}:=B_{i}+t_{i} M_{i}\right)$ has an lc centre of dimension one contained in Supp $M_{i}$, then it is quite easy to get a contradiction. We may then assume that each $\left(X_{i}, \Delta_{i}\right)$ has an lc centre $P_{i}$ of dimension zero contained in Supp $M_{i}$. We may also assume that $\left(X_{i}, \Delta_{i}\right)$ is plt outside $P_{i}$. Let $\left(Y_{i}, \Delta_{Y_{i}}\right)$ be a $\mathbb{Q}$-factorial dlt model of $\left(X_{i}, \Delta_{i}\right)$ such that there are some exceptional divisors on $Y_{i}$ mapping to $P_{i}$. Such $Y_{i}$ exist by a version of Lemma 7.7 in dimension 2 .

There is a prime exceptional divisor $E_{i}$ of $Y_{i} \rightarrow X_{i}$ such that it intersects the birational transform of $M_{i}$. Note that $E_{i}$ is normal and actually isomorphic to $\mathbb{P}_{k}^{1}$ since $E_{i}$ is a component of $\left\lfloor\Delta_{Y_{i}}\right\rfloor$ and $\left(K_{Y_{i}}+\Delta_{Y_{i}}\right) \cdot E_{i}=0$. Now by adjunction define $K_{E_{i}}+\Delta_{E_{i}}=\left.\left(K_{Y_{i}}+\Delta_{Y_{i}}\right)\right|_{E_{i}}$. Then by Proposition 4.2 and its proof, the set of all the coefficients of the $\Delta_{E_{i}}$ is a subset of a fixed DCC set but they do not satisfy ACC. This is a contradiction since $\operatorname{deg} \Delta_{E_{i}}=2$.

We apply the ACC of 11.2 to negativity of contractions. 
Lemma 11.3. Let $\Lambda \subset[0,1]$ be a DCC set of real numbers. Then there is $\epsilon>0$ satisfying the following: assume we have

- a klt pair $(X, B)$ of dimension 2 ,

- the coefficients of $B$ belong to $\Lambda \cup[1-\epsilon, 1]$,

- $f: X \rightarrow Y$ is an extremal birational projective contraction with exceptional divisor $E$,

- the coefficient of $E$ in $B$ belongs to $[1-\epsilon, 1]$, and

- $-\left(K_{X}+B\right)$ is nef/Y.

If $\Delta$ is obtained from $B$ by replacing each coefficient in $[1-\epsilon, 1]$ with 1 , then $-\left(K_{X}+\Delta\right)$ is also nef/Y.

Proof. Note that klt pairs of dimension 2 are $\mathbb{Q}$-factorial so $K_{X}+\Delta$ is $\mathbb{R}$-Cartier. By 11.2, we can pick $\epsilon>0$ so that: if $(T, C)$ is lc of dimension 2 and $M \geq 0$ such that the coefficients of $C$ belong to $\Lambda$ and the coefficients of $M$ belong to $\{1\}$, then the lc threshold $\operatorname{lct}(M, T, C)$ does not belong to $[1-\epsilon, 1)$.

Now since $(X, B)$ is klt and $-\left(K_{X}+B\right)$ is nef $/ Y,\left(Y, B_{Y}\right)$ is also klt. Thus $\left(Y, \Delta_{Y}-\epsilon\left\lfloor\Delta_{Y}\right\rfloor\right)$ is klt because $B_{Y} \geq \Delta_{Y}-\epsilon\left\lfloor\Delta_{Y}\right\rfloor$. In particular, the lc threshold of $\left\lfloor\Delta_{Y}\right\rfloor$ with respect to $\left(Y, \Delta_{Y}-\left\lfloor\Delta_{Y}\right\rfloor\right)$ is at least $1-\epsilon$. Note that the coefficients of $\Delta$ belong $\Lambda \cup\{1\}$ and the coefficients of $\Delta_{Y}-\left\lfloor\Delta_{Y}\right\rfloor$ belong to $\Lambda$. Thus by our choice of $\epsilon$, the pair $\left(Y, \Delta_{Y}\right)$ is lc. Therefore we can write

$$
K_{X}+\Delta=f^{*}\left(K_{Y}+\Delta_{Y}\right)+e E
$$

for some $e \geq 0$ because the coefficient of $E$ in $\Delta$ is 1 . This implies that $-\left(K_{X}+\Delta\right)$ is indeed nef $/ Y$.

11.4. Global ACC for surfaces. In this subsection we prove a global type of ACC for surfaces (11.7) which will be used in the proof of Theorem 1.10.

Construction 11.5 Let $\epsilon \in(0,1)$ and let $X^{\prime}$ be a klt Fano surface with $\rho\left(X^{\prime}\right)=1$. Assume that $X^{\prime}$ is not $\epsilon$-lc. Pick a prime divisor $E$ (on birational models of $\left.X^{\prime}\right)$ with $\log$ discrepancy $a\left(E, X^{\prime}, 0\right)<\epsilon$. By a version of Lemma 7.7 in dimension two, there is a birational contraction $Y^{\prime} \rightarrow X^{\prime}$ which is extremal and has $E$ as the only exceptional divisor. Under our assumptions it is easy to find a boundary $D_{Y^{\prime}}$ such that $\left(Y^{\prime}, D_{Y^{\prime}}\right)$ is klt and $K_{Y^{\prime}}+D_{Y^{\prime}} \sim_{\mathbb{R}}-e E$ for some $e>0$. In particular, we can run an LMMP on $-E$ which ends with a Mori fibre space $X^{\prime \prime} \rightarrow T^{\prime \prime}$ so that $E^{\prime \prime}$ positively intersects the extremal ray defining $X^{\prime \prime} \rightarrow T^{\prime \prime}$ where $E^{\prime \prime}$ is the birational transform of $E$.

As $\rho\left(X^{\prime}\right)=1$, we get $\rho\left(Y^{\prime}\right)=2$. One of the extremal rays of $Y^{\prime}$ gives the contraction $Y^{\prime} \rightarrow X^{\prime}$. The other one either gives $X^{\prime \prime} \rightarrow T^{\prime \prime}$ with $Y^{\prime}=X^{\prime \prime}$ or it gives a birational contraction $Y^{\prime} \rightarrow X^{\prime \prime}$. If $\operatorname{dim} T^{\prime \prime}=0$, then $X^{\prime \prime}$ is also a klt Fano with $\rho\left(X^{\prime \prime}\right)=1$.

Lemma 11.6. Let $b \in(0,1)$ be a real number. Then there is a natural number $m$ depending only on $b$ such that: let $(X, B)$ be a klt pair of dimension 2 and $x \in X$ a closed point; then the number of those components of $B$ containing $x$ and with coefficient $\geq b$ is at most $m$. 


\section{Existence of flips and minimal models for 3-folds in char $p \quad 43$}

Proof. Since $(X, B)$ is klt and $\operatorname{dim} X=2, X$ is $\mathbb{Q}$-factorial. We can assume that each coefficient of $B$ is equal to $b$ by discarding any component with coefficient less than $b$ and by decreasing each coefficient which is more than $b$. Moreover, we can assume every component of $B$ contains $x$.

Pick a nonzero $\mathbb{R}$-Cartier divisor $G \geq 0$ such that $(X, C:=B+G)$ is lc near $x$ and such that $x$ is a lc centre of $(X, B+G)$ : for example we can take a $\log$ resolution $W \rightarrow X$ and let $G$ be the pushdown of an appropriate ample $\mathbb{R}$-divisor on $W$. Shrinking $X$ we can assume $(X, C)$ is lc. Since $(X, B)$ is klt, there is an extremal contraction $f: Y \rightarrow X$ which extracts a prime divisor $S$ with log discrepancy $a(S, X, C)=0$.

Let $B_{Y}$ be the sum of $S$ and the birational transform of $B$. Then $-\left(K_{Y}+B_{Y}\right)$ is ample/X. Apply adjunction (4.2) and write $K_{S^{\nu}}+B_{S^{\nu}}$ for the pullback of $K_{Y}+B_{Y}$ to the normalization of $S$. As $-\left(K_{S^{\nu}}+B_{S^{\nu}}\right)$ is ample, $S^{\nu} \simeq \mathbb{P}^{1}$ and $\operatorname{deg} B_{S^{\nu}}<2$.

By 4.2, the coefficient of each $s \in \operatorname{Supp} B_{S^{\nu}}$ is of the form $\frac{n-1}{n}+\frac{r b}{n}$ for some integer $r \geq 0$ and some $n \in \mathbb{N} \cup\{\infty\}$. In particular, the number of the components of $B_{S^{\nu}}$ is bounded and the number $r$ in the formula is also bounded. This bounds the number of the components of $B$ because $r$ is more than or equal to the number of those components of $B_{Y}-S$ which pass through the image of $s$.

Proposition 11.7. Let $\Lambda \subset[0,1]$ be a DCC set of real numbers. Then there is a finite subset $\Gamma \subset \Lambda$ with the following property: let $(X, B)$ be a pair and $X \rightarrow Z$ a projective morphism such that

- $(X, B)$ is lc of dimension 2 over $k$,

- the coefficients of $B$ are in $\Lambda$,

- $K_{X}+B \equiv 0 / Z$,

- $\operatorname{dim} X>\operatorname{dim} Z$.

Then the coefficient of each horizontal/Z component of $B$ is in $\Gamma$.

Proof. Step 1. We can assume that $1 \in \Lambda$. If the proposition is not true, then there is a sequence $\left(X_{i}, B_{i}\right), X_{i} \rightarrow Z_{i}$ of pairs and morphisms as in the proposition such that the set of the coefficients of the horizontal $/ Z_{i}$ components of all the $B_{i}$ put together does not satisfy ACC. By taking $\mathbb{Q}$-factorial dlt models we can assume that $\left(X_{i}, B_{i}\right)$ are $\mathbb{Q}$-factorial dlt. Write $B_{i}=\sum b_{i, j} B_{i, j}$. We may assume that $B_{i, 1}$ is horizontal $/ Z_{i}$ and that $b_{1,1}<b_{2,1}<\cdots$.

Step 2. First assume that $\operatorname{dim} Z_{i}=1$ for every $i$. Run the $\operatorname{LMMP} / Z_{i}$ on $K_{X_{i}}+B_{i}-b_{i, 1} B_{i, 1}$ with scaling of $b_{i, 1} B_{i, 1}$. This terminates with a model $X_{i}^{\prime}$ having an extremal contraction $X_{i}^{\prime} \rightarrow Z_{i}^{\prime} / Z_{i}$ such that $K_{X_{i}^{\prime}}+B_{i}^{\prime}-b_{i, 1} B_{i, 1}^{\prime}$ is numerically negative over $Z_{i}^{\prime}$. Let $F_{i}^{\prime}$ be the reduced variety associated to a general fibre of $X_{i}^{\prime} \rightarrow Z_{i}^{\prime}$. Since $K_{X_{i}^{\prime}}+B_{i}^{\prime} \equiv 0 / Z^{\prime}$ and ${F_{i}^{\prime}}^{2}=0$, we get $\left(K_{X_{i}^{\prime}}+\right.$ $\left.B_{i}^{\prime}+F_{i}^{\prime}\right) \cdot F_{i}^{\prime}=0$ hence the arithmetic genus $p_{a}\left(F_{i}^{\prime}\right)<0$ which implies that $F_{i}^{\prime} \simeq \mathbb{P}_{k}^{1}$. We can write

$$
\left.\operatorname{deg}\left(K_{X_{i}^{\prime}}+B_{i}^{\prime}+F_{i}^{\prime}\right)\right|_{F_{i}^{\prime}}=-2+\sum n_{i, j} b_{i, j}=0
$$


for certain integers $n_{i, j} \geq 0$ such that $n_{i, 1}>0$. Since the $b_{i, j}$ belong to the DCC set $\Lambda, n_{i, 1}$ is bounded from above and below. Moreover, we can assume that the sums $\sum_{j \geq 2} n_{i, j} b_{i, j}$ satisfy the DCC hence $n_{i, 1} b_{i, 1}=2-\sum_{j \geq 2} n_{i, j} b_{i, j}$ satisfies the ACC, a contradiction.

Step 3. From now on we may assume that $\operatorname{dim} Z_{i}=0$ for every $i$. Run the $\mathrm{LMMP} / Z_{i}$ on $K_{X_{i}}+B_{i}-b_{i, 1} B_{i, 1}$ with scaling of $b_{i, 1} B_{i, 1}$. This terminates with a model $X_{i}^{\prime}$ having an extremal contraction $X_{i}^{\prime} \rightarrow Z_{i}^{\prime}$ such that $K_{X_{i}^{\prime}}+B_{i}^{\prime}-b_{i, 1} B_{i, 1}^{\prime}$ is numerically negative over $Z_{i}^{\prime}$. If $\operatorname{dim} Z_{i}^{\prime}=1$ for infinitely many $i$, then we get a contradiction by Step 2. So we assume that $Z_{i}^{\prime}$ are all points hence each $X_{i}^{\prime}$ is a Fano with Picard number one.

Assume that $\left(X_{i}{ }^{\prime}, B_{i}^{\prime}\right)$ is lc but not klt for every $i$. Assume that each $\left(X_{i}{ }^{\prime}, B_{i}^{\prime}\right)$ has an lc centre $S_{i}^{\prime}$ of dimension one. Let $K_{S_{i}^{\prime}}+B_{S_{i}^{\prime}}=\left.\left(K_{X_{i}^{\prime}}+B_{i}^{\prime}\right)\right|_{S_{i}^{\prime}}$ by adjunction. Note that $S_{i}^{\prime}$ is normal since $\left(X_{i}^{\prime}, B_{i}^{\prime}-b_{i, 1} B_{i, 1}^{\prime}\right)$ is $\mathbb{Q}$-factorial dlt. Since $K_{S_{i}^{\prime}}+B_{S_{i}^{\prime}} \equiv 0, S_{i}^{\prime} \simeq \mathbb{P}_{k}^{1}$. If $\operatorname{Supp} B_{i, 1}^{\prime}$ contains an lc centre for infinitely many $i$, then we get a contradiction by ACC for lc thresholds in dimension 2. So we can assume that Supp $B_{i, 1}^{\prime}$ does not contain any lc centre, in particular, none of the points of $S_{i}^{\prime} \cap B_{i, 1}^{\prime}$ is an lc centre. Now, since $\left\{b_{i, j}\right\}$ does not satisfy ACC, by Proposition 4.2, the set of the coefficients of all the $B_{S_{i}^{\prime}}$ satisfies DCC but not ACC which gives a contradiction as above (by considering the coefficients of the points in $\left.S_{i}^{\prime} \cap B_{i, 1}^{\prime}\right)$. So we can assume that each $\left(X_{i}{ }^{\prime}, B_{i}^{\prime}\right)$ has an lc centre of dimension zero. By a version of Lemma 7.7 in dimension 2, there is a projective birational contraction $Y_{i}^{\prime} \rightarrow X_{i}^{\prime}$ which extracts only one prime divisor $E_{i}^{\prime}$ and it satisfies $a\left(E_{i}^{\prime}, X_{i}^{\prime}, B_{i}^{\prime}\right)=0$. Let $K_{Y_{i}^{\prime}}+B_{Y_{i}^{\prime}}$ be the pullback of $K_{X_{i}^{\prime}}+B_{i}^{\prime}$. By running the LMMP on $K_{Y_{i}^{\prime}}+B_{Y_{i}^{\prime}}-E_{i}^{\prime}$, we arrive on a model on which either the birational transform of $E_{i}^{\prime}$ intersects the birational transform of $B_{i, 1}^{\prime}$ for infinitely many $i$, or we get a Mori fibre space over a curve whose general fibre intersects the birational transform of $B_{i, 1}^{\prime}$ for infinitely many $i$. In any case, we can apply the arguments above to get a contradiction. So from now on we may assume that $\left(X_{i}^{\prime}, B_{i}^{\prime}\right)$ are all klt.

Step 4 . If there is $\epsilon>0$ such that $X_{i}^{\prime}$ is $\epsilon$-lc for every $i$, then we are done since such $X_{i}^{\prime}$ are bounded by Alexeev [1]. So we can assume that the minimal log discrepancies of the $X_{i}^{\prime}$ form a strictly decreasing sequence of positive numbers. Since $\left(X_{i}^{\prime}, B_{i}^{\prime}\right)$ are klt, we can assume that the minimal log discrepancies of the $\left(X_{i}^{\prime}, B_{i}^{\prime}\right)$ also form a strictly decreasing sequence of positive numbers. As in Construction 11.5, we find a contraction $Y_{i}^{\prime} \rightarrow X_{i}^{\prime}$ extracting a prime divisor $E_{i}$ with minimal log discrepancy $a\left(E_{i}, X_{i}^{\prime}, B_{i}^{\prime}\right)<\epsilon$ and run a $-E_{i}$-LMMP to get a Mori fibre structure $X_{i}^{\prime \prime} \rightarrow Z_{i}^{\prime \prime}$. If $\operatorname{dim} Z_{i}^{\prime \prime}=1$ for each $i$, we use Step 2 to get a contradiction. So we may assume that $\operatorname{dim} Z_{i}^{\prime \prime}=0$ for each $i$. Note that the exceptional divisor of $X_{i}^{\prime \prime} \rightarrow-\rightarrow X_{i}^{\prime}$ is a component of $B_{i}^{\prime \prime}$ with coefficient $\geq 1-\epsilon$ where $K_{X_{i}^{\prime \prime}}+B_{i}^{\prime \prime}$ is the pullback of $K_{X_{i}^{\prime}}+B_{i}^{\prime}$.

Write $K_{Y_{i}^{\prime}}+B_{Y_{i}^{\prime}}$ for the pullback of $K_{X_{i}^{\prime}}+B_{i}^{\prime}$. By construction, the coefficients of $B_{Y_{i}^{\prime}}$ belong to some DCC subset of $\Lambda \cup[1-\epsilon, 1]$. We show that if $\epsilon$ is sufficiently small, then $Y_{i}^{\prime} \rightarrow X_{i}^{\prime \prime}$ cannot contract a component of $B_{Y_{i}^{\prime}}$ with coefficient $\geq 1-\epsilon$. Indeed let $\Delta_{Y_{i}^{\prime}}$ be obtained from $B_{Y_{i}^{\prime}}$ by replacing each coefficient $\geq 1-\epsilon$ with 1 . Then by Lemma $11.3,-\left(K_{Y_{i}^{\prime}}+\Delta_{Y_{i}^{\prime}}\right)$ is nef over both 
Existence of flips and minimal models for 3-folds in char $p \quad 45$

$X_{i}^{\prime}$ and $X_{i}^{\prime \prime}$. As $\rho\left(Y_{i}^{\prime}\right)=2,-\left(K_{Y_{i}^{\prime}}+\Delta_{Y_{i}^{\prime}}\right)$ is nef globally. This is a contradiction because the pushdown of $K_{Y_{i}^{\prime}}+\Delta_{Y_{i}^{\prime}}$ to $X_{i}^{\prime \prime}$ is ample.

Step 5. Now replace $\left(X_{i}^{\prime}, B_{i}^{\prime}\right)$ with $\left(X_{i}^{\prime \prime}, B_{i}^{\prime \prime}\right)$ and repeat the process of Step $4, m$ times. By the last paragraph the new components of $B_{i}^{\prime}$ that appear in the process are not contracted again. So we may assume that we have at least $m$ components of $B_{i}^{\prime}$ with coefficients $\geq 1-\epsilon$. Let $x_{i}^{\prime}$ be the image of the exceptional divisor of $Y_{i}^{\prime} \rightarrow X_{i}^{\prime}$ and let $x_{i}^{\prime \prime}$ be the image of the exceptional divisor of $Y_{i}^{\prime} \rightarrow X_{i}^{\prime \prime}$. Also let $m_{i}^{\prime}$ be the number of those components of $B_{i}^{\prime}$ with coefficient $\geq 1-\epsilon$ and passing through $x_{i}^{\prime}$. Define $m_{i}^{\prime \prime}$ similarly. Since $\rho\left(Y_{i}^{\prime}\right)=2$, each component of $B_{Y_{i}^{\prime}}$ intersects the exceptional divisor of $Y_{i}^{\prime} \rightarrow X_{i}^{\prime}$ or the exceptional divisor of $Y_{i}^{\prime} \rightarrow X_{i}^{\prime \prime}$. Therefore, $m_{i}^{\prime}+m_{i}^{\prime \prime} \geq m$.

Finally by Lemma 11.6 both $m_{i}^{\prime}$ and $m_{i}^{\prime \prime}$ are bounded hence $m$ is also bounded. This means that after finitely many times applying the process of Step 4, we can assume there is $\epsilon>0$ such that $X_{i}^{\prime}$ is $\epsilon$-lc for every $i$, and then apply boundedness of such $X_{i}^{\prime}[1]$.

\subsection{3-folds.}

Proof. (of Theorem 1.10) If the theorem does not hold, then there is a sequence $\left(X_{i}, B_{i}\right)$ of lc pairs of dimension 3 over $k$ and $\mathbb{R}$-Cartier divisors $M_{i} \geq 0$ such that the coefficients of $B_{i}$ are in $\Lambda$, the coefficients of $M_{i}$ are in $\Gamma$ but such that the $t_{i}:=\operatorname{lct}\left(M_{i}, X_{i}, B_{i}\right)$ form a strictly increasing sequence of numbers. We may assume that each $\left(X_{i}, \Delta_{i}:=B_{i}+t_{i} M_{i}\right)$ has an lc centre of dimension $\leq 1$ contained in Supp $M_{i}$. Let $\left(Y_{i}, \Delta_{Y_{i}}\right)$ be a $\mathbb{Q}$-factorial dlt model of $\left(X_{i}, \Delta_{i}\right)$ such that there is an exceptional divisor on $Y_{i}$ mapping onto an lc centre inside Supp $M_{i}$. Such $Y_{i}$ exist by Lemma 7.7.

There is a prime exceptional divisor $E_{i}$ of $Y_{i} \rightarrow X_{i}$ such that it intersects the birational transform of $M_{i}$ and that it maps into Supp $M_{i}$. Note that $E_{i}$ is normal by Lemma 5.2. Let $E_{i} \rightarrow Z_{i}$ be the contraction induced by $E_{i} \rightarrow X_{i}$. Now by adjunction define $K_{E_{i}}+\Delta_{E_{i}}=\left.\left(K_{Y_{i}}+\Delta_{Y_{i}}\right)\right|_{E_{i}}$. Then the set of all the coefficients of the horizontal $/ Z_{i}$ components of the $\Delta_{E_{i}}$ satisfies DCC but not ACC, by Proposition 4.2. This contradicts Proposition 11.7.

\section{Non-BIG LOG DIVISORS: PROOF OF 1.11}

Lemma 12.1. Let $X$ be a normal projective variety of dimension $d$ over an algebraically closed field (of any characteristic). Let $A$ an ample $\mathbb{R}$-divisor and $P$ a nef $\mathbb{R}$-divisor with $P^{d}=0$. Then for any $\epsilon>0$, there exist $\delta \in[0, \epsilon]$ and a very ample divisor $H$ such that $(P-\delta A) \cdot H^{d-1}=0$.

Proof. First we show that there is an ample divisor $H$ such that $(P-\epsilon A) \cdot H^{d-1}<$ 0 . Put $r(\tau):=(P-\epsilon A)(P+\tau A)^{d-1}$. Then

$$
r(\tau)=(P-\epsilon A)\left(P^{d-1}+a_{d-2} \tau P^{d-2} A+\cdots+a_{1} \tau^{d-2} P A^{d-2}+\tau^{d-1} A^{d-1}\right)
$$


where the $a_{i}>0$ depend only on $d$. Put $a_{d-1}=a_{0}=1, a_{-1}=0$, and let $n$ be the smallest integer such that $P^{d-n} A^{n} \neq 0$. Then we can write

$$
r(\tau)=\sum_{i=0}^{d-1}\left(a_{i-1} \tau^{d-i}-\epsilon a_{i} \tau^{d-i-1}\right) P^{i} A^{d-i}
$$

from which we get

$$
r(\tau)=\sum_{i=0}^{d-n}\left(a_{i-1} \tau^{d-i}-\epsilon a_{i} \tau^{d-i-1}\right) P^{i} A^{d-i}
$$

hence

$$
\frac{r(\tau)}{\tau^{n-1}}=\left(a_{d-n-1} \tau-\epsilon a_{d-n}\right) P^{d-n} A^{n}+\tau s(\tau)
$$

for some polynomial function $s(\tau)$. Now if $\tau>0$ is sufficiently small it is clear that the right hand side is negative hence $r(\tau)<0$.

Choose $\tau>0$ so that $r(\tau)<0$. Since $P+\tau A$ is ample and ampleness is an open condition, there is an ample $\mathbb{Q}$-divisor $H$ close to $P+\tau A$ such that $(P-\epsilon A) \cdot H^{d-1}<0$. By replacing $H$ with a multiple we can assume that $H$ is very ample. Since $P \cdot H^{d-1} \geq 0$ by the nefness of $P$, it is then obvious that there is some $\delta \in[0, \epsilon]$ such that $(P-\delta A) \cdot H^{d-1}=0$.

Proof. (of Theorem 1.11) Assume that $D^{d}=0$. By replacing $A$ we may assume that it is ample. Fix $\alpha>0$. By Lemma 12.1, there exist a number $t$ sufficiently close to 1 (possibly equal to 1 ) and a very ample divisor $H$ such that

$$
\left(K_{X}+B+t(A+\alpha D)\right) \cdot H^{d-1}=0
$$

Now we can view $H^{d-1}$ as a 1 -cycle on $X$. For each point $x \in X$, there is an effective 1-cycle $C_{x}$ whose class is the same as $H^{d-1}$ and such that $x \in C_{x}$. Since $H$ is very ample, we may assume that $C_{x}$ is irreducible and that it is inside the smooth locus of $X$ for general $x$. In particular, we have

$$
\left(K_{X}+B+t(A+\alpha D)\right) \cdot C_{x}=0
$$

Pick a general $x \in X$ and let $C_{x}$ be the curve mentioned above. Since $B$ is effective and $A+\alpha D$ is ample, we get $K_{X} \cdot C_{x}<0$. Thus by Kollár [19, Chapter II, Theorem 5.8], there is a rational curve $L_{x}$ passing through $x$ such that

$$
\begin{aligned}
0<A \cdot L_{x} & \leq(A+\alpha D) \cdot L_{x} \leq(2 d) \frac{(A+\alpha D) \cdot C_{x}}{-K_{X} \cdot C_{x}} \\
= & \frac{2 d}{t}\left(1+\frac{B \cdot C_{x}}{K_{X} \cdot C_{x}}\right) \leq \frac{2 d}{t}<3 d
\end{aligned}
$$

because $K_{X} \cdot C_{x}<0, B \cdot C_{x} \geq 0$, and $t$ is sufficiently close to 1 . Note that although $K_{X}$ and $B$ need not be $\mathbb{R}$-Cartier, the intersection numbers still make sense since $C_{x}$ is inside the smooth locus of $X$.

As $A$ is ample and $A \cdot L_{x} \leq 3 d$, we can assume that such $L_{x}$ (for general $x$ ) belong to a bounded family $\mathcal{L}$ of curves on $X$ (independent of the choice of $t, \alpha$ ). Therefore there are only finitely many possibilities for the intersection numbers 


\section{Existence of flips and minimal models for 3-folds in char $p \quad 47$}

$D \cdot L_{x}$. If we choose $\alpha$ sufficiently large, then the inequality $(A+\alpha D) \cdot L_{x} \leq 3 d$ implies $D \cdot L_{x}=0$ and so we get the desired family.

\section{REFERENCES}

[1] V. Alexeev; Boundedness and $K^{2}$ for log surfaces. Internat. J. Math. 5 (1994), no. 6, 779-810.

[2] C. Birkar; On existence of log minimal models and weak Zariski decompositions. Math Annalen, Volume 354 (2012), Number 2, 787-799.

[3] C. Birkar; On existence of log minimal models. Compositio Math. volume 146 (2010), 919-928.

[4] C. Birkar; Ascending chain condition for lc thresholds and termination of log flips. Duke math. Journal, volume 136, no. 1 (2007), 173-180.

[5] C. Birkar, P. Cascini, C. D. Hacon, J. Mckernan; Existence of minimal models for varieties of log general type. J. Amer. Math. Soc. 23 (2010), 405-468.

[6] C. Birkar, M. Păun; Minimal models, flips and finite generation : a tribute to V.V. Shokurov and Y.-T. Siu. In "Classification of algebraic varieties", European Math Society series of congress reports (2010).

[7] P. Cascini, Y. Gongyo, K. Schwede; Uniform bounds for strongly F-regular surfaces. arXiv:1402.0027v2.

[8] P. Cascini, J. M Kernan, M. Mustaţă; The augmented base locus in positive characteristic. Proceedings of the Edinburgh Mathematical Society (Series 2), Volume 57, Issue 01, (2014), 79-87.

[9] P. Cascini, H. Tanaka, C. Xu; On base point freeness in positive characteristic. arXiv:1305.3502v1.

[10] V. Cossart and O. Piltant; Resolution of singularities of threefolds in positive characteristic II. Journal of Algebra 321 (2009), 1836-1976.

[11] V. Cossart and O. Piltant; Resolution of singularities of threefolds in positive characteristic I. J. Algebra 320 (2008), 1051-1082.

[12] S. D. Cutkosky; Resolution of singularities for 3-folds in positive characteristic. American Journal of Mathematics, Volume 131, Number 1, (2009), 59-127.

[13] C. Hacon and C. Xu; On the three dimensional minimal model program in positive characteristic. To appear in J. Amer. Math. Soc., arXiv:1302.0298v2.

[14] Y. Kawamata; Semistable minimal models of threefolds in positive or mixed characteristic. J. Alg. Geom. 3 (1994), 463-491.

[15] Y. Kawamata, K. Matsuda, K. Matsuki; Introduction to the minimal model problem. Algebraic geometry (Sendai, 1985), Adv. Stud. Pure Math., no. 10, North-Holland, Amsterdam (1987), 283-360.

[16] S. Keel; Basepoint freeness for nef and big line bundles in positive characteristic. Annals of Math, Second Series, Vol. 149, No. 1 (1999), 253-286.

[17] S. Keel, K. Matsuki, J. M'Kernan; Log abundance theorem for threefolds. Duke Math. J. Volume 75, Number 1 (1994), 99-119.

[18] J. Kollár; Singularities of the minimal model program. Cambridge University Press (2013).

[19] J. Kollár; Rational curves on algebraic varieties. Springer, 1999 edition.

[20] J. Kollár; Flips and abundance for algebraic threefolds. Astérisque 211, Soc. Math. France, 1992.

[21] J. Kollár, S. Mori; Birational Geometry of Algebraic Varieties. Cambridge University Press (1998).

[22] J. McKernan, Yu. Prokhorov; Threefold thresholds. Manuscripta Math. 114 (2004), no. $3,281-304$. 
[23] I.R. Shafarevich; Lectures on minimal models and birational transformations of two dimensional schemes. Notes by C. P. Ramanujam. Tata Institute of Fundamental Research Lectures on Mathematics and Physics, Vol. 37. (1966).

[24] K. Schwede; F-singularities and Frobenius splitting. Lecture notes. Available on the authors's website.

[25] V.V. Shokurov; Prelimiting flips. Proc. Steklov Inst. Math. 240 (2003), 75-213.

[26] V.V. Shokurov; Three-dimensional log flips. With an appendix in English by Yujiro Kawamata. Russian Acad. Sci. Izv. Math. 40 (1993), no. 1, 95-202.

[27] V. V. Shokurov; The nonvanishing theorem. Izv. Akad. Nauk SSSR Ser. Mat., 49:3 (1985), 635-651.

[28] H. Tanaka; Minimal models and abundance for positive characteristic log surfaces. To appear in Nagoya Math. J., arXiv:1201.5699v2.

[29] H. Tanaka; Abundance theorem for semi log canonical surfaces in positive characteristic. arXiv: $1301.6889 \mathrm{v} 1$

[30] C. Xu; On base point free theorem of threefolds in positive characteristic. arXiv:1311.3819v2.

DPMMS, Centre for Mathematical Sciences, Cambridge University,

Wilberforce Road,

Cambridge, CB3 0WB,

UK

email: c.birkar@dpmms.cam.ac.uk 